Received: 23 October 2017

Accepted: 8 January 2018

Published online: 22 January 2018

\section{New functions of Semaphorin 3E and its receptor PlexinD1 during developing and adult hippocampal formation}

\author{
Agata Mata ${ }^{1,2,3,4}$, Vanessa Gil ${ }^{1,2,3,4}$, Jeús Pérez-Clausell ${ }^{2}$, Miguel Dasilva ${ }^{5}$, Mari Carmen \\ González-Calixto ${ }^{7}$, Eduardo Soriano $2,3,4,6,8$, José Manuel García-Verdugo ${ }^{7}$, Maria \\ V. Sanchez-Vives $\mathbb{D}^{5,6}$ \& José Antonio del Río $\mathbb{1 D}^{1,2,3,4}$
}

The development and maturation of cortical circuits relies on the coordinated actions of long and short range axonal guidance cues. In this regard, the class 3 semaphorins and their receptors have been seen to be involved in the development and maturation of the hippocampal connections. However, although the role of most of their family members have been described, very few data about the participation of Semaphorin 3E (Sema3E) and its receptor PlexinD1 during the development and maturation of the entorhino-hippocampal (EH) connection are available. In the present study, we focused on determining their roles both during development and in adulthood. We determined a relevant role for Sema3E/ PlexinD1 in the layer-specific development of the EH connection. Indeed, mice lacking Sema3E/ PlexinD1 signalling showed aberrant layering of entorhinal axons in the hippocampus during embryonic and perinatal stages. In addition, absence of Sema3E/PlexinD1 signalling results in further changes in postnatal and adult hippocampal formation, such as numerous misrouted ectopic mossy fibers. More relevantly, we describe how subgranular cells express PlexinD1 and how the absence of Sema3E induces a dysregulation of the proliferation of dentate gyrus progenitors leading to the presence of ectopic cells in the molecular layer. Lastly, Sema3E mutant mice displayed increased network excitability both in the dentate gyrus and the hippocampus proper.

The hippocampal formation plays crucial roles in the consolidation of information from short- to long-term memory, as well as in spatial memory ${ }^{1,2}$. Since different neuronal cell types, the main extrinsic afferent connections (i.e., entorhinal or commissural/associational fibers) and the most relevant intrinsic connection (i.e., the mossy fibers) are organized into well-defined lamina in the hippocampus ${ }^{3}$, it has frequently been used as a system model for studying fundamental neuroscience including neurophysiology ${ }^{4}$. During axonal wiring in perinatal development in rodents, entorhinal axons refrain from invading the adjacent ventrolateral isocortex, entering the hippocampal formation to reach the stratum lacunosum moleculare (slm) of the hippocampus proper to further innervate the outermost portion of the molecular layer (oml) of the fascia dentata ${ }^{5-8}$. In addition, axons from isocortical regions close to the entorhinal cortex (ventrolateral neocortex) avoid the hippocampal region (including hippocampal and retrohippocampal formation) (e.g. ${ }^{7}$ ). In fact, based on classical neuroanatomical studies using axonal tracers (e.g. $\left.{ }^{5-7}\right)$, cell transplantation in vivo (e.g. ${ }^{9-11}$ ) and in vitro in slices (e.g. ${ }^{12-17}$ ), it has been suggested that axons from entorhinal neurons are able to reach specifically the slm/oml of the hippocampal formation in healthy conditions. In fact, commissural/associational axons avoid the $\mathrm{slm} / \mathrm{oml}$ in $v i v o^{5-7,18}$, as well

\footnotetext{
${ }^{1}$ Molecular and Cellular Neurobiotechnology, Institute for Bioengineering of Catalonia (IBEC), The Barcelona Institute of Science and Technology, Parc Científic de Barcelona, Barcelona, Spain. '2Department of Cell Biology, Physiology and Immunology, Universitat de Barcelona, Barcelona, Spain. ${ }^{3}$ Centro de Investigación Biomédica en Red sobre Enfermedades Neurodegenerativas, (CIBERNED), Barcelona, Spain. ${ }^{4}$ Institut de Neurociències de la Universitat de Barcelona, Barcelona, Spain. ${ }^{5}$ Systems Neuroscience, Institut d'Investigacions Biomèdiques August Pi i Sunyer (IDIBAPS), Barcelona, Spain. ${ }^{6}$ ICREA, Barcelona, Spain. ${ }^{7}$ Laboratory of Comparative Neurobiology, Institute Cavanilles, University of Valencia, CIBERNED, 46980, Valencia, Spain. ${ }^{8}$ Vall d'Hebrón Institut de Recerca (VHIR), Barcelona, Spain. Agata Mata and Vanessa Gil contributed equally to this work. Correspondence and requests for materials should be addressed to J.A.R. (email: jadelrio@ibecbarcelona.eu)
} 
as in vitro ${ }^{16,19,20}$, being restricted to the stratum radiatum of the hippocampus proper and the innermost portion of the molecular layer (iml) of the dentate gyrus ${ }^{8}$. With respect to mossy fibers, postnatal and adult newborn granule cells extend their axons, forming synaptic contacts on hilar mossy cells and on proximal dendrites of the CA3 pyramidal cells in the stratum lucidum ${ }^{3,8}$.

Axonal specification at the hippocampus is a highly orchestrated process regulated by a large number of factors, both at the topographic and local levels, that have been sequentially revealed for years (e.g. ${ }^{21-23}$ ). One of these molecule families, semaphorins and their receptors neuropilins as well as their co-receptors, plexins, have emerged as important cellular cues regulating key developmental processes, such as neuronal migration and axonal guidance ${ }^{24-26}$. In particular, during hippocampal development, the participation of class III semaphorins in the ingrowth and maturation of entorhino-hippocampal (EH) (mainly Sema3A and 3F), septo-hippocampal (Sema3C) or subicular connections (Sema3E) has been described ${ }^{27-32}$. In addition, Sema3F together with its receptor complex Neuropilin2/PlexinA3 actively participate in the maturation of the hippocampal mossy fib$\mathrm{ers}^{33-35}$. However, the participation of Sema3E and its receptor PlexinD1 in the development of the hippocampal connections has not yet been analysed in detail, and differing data have been published. Indeed, Pozas et al., showed that in mice Sema3E repels exclusively hippocampal axons but only at embryonic day 14.5 (E14.5) without effects from E14.5 onwards ${ }^{30}$. This was not observed by Chauvet et al., and Deck et al., who found that Sema3E is able to collapse cortical axons around E17. $5^{32}$ or E14.5-16.5 $5^{36}$, respectively.

Functions of Sema3E/PlexinD1 are also associated with vascular development and remodelling, and axon regeneration after injury or cancer (e.g. ${ }^{37-42}$ ). Indeed, as described for other signalling mechanisms (i.e., Netrins ${ }^{43}$ ), Sema3E/PlexinD1 might play several roles in different cell types during development and in the adult. Keeping this in mind, in the present study we analyse in detail the participation of Sema3E and its receptor PlexinD1 during development and in adult hippocampal region. We focus our experiments on analysis of the afferent connection (EH-connection) during development as well as on the correlation of the putative changes with the laminar distribution of hippocampal afferents in the adult. Moreover, we explore cell-specific functions of Sema3E/PlexinD1 in the hippocampal formation. Considering that semaphorins have been described as being involved in cell proliferation (e.g. ${ }^{44,45}$ ), we focus our attention on analysing whether absence of Sema3E-mediated signalling modifies cell proliferation in the adult hippocampus and putative effects on mossy fiber growth and hippocampal physiology. Our results indicate that Sema3E and PlexinD1 participate actively in the establishment of the $\mathrm{EH}$ connection by regulating the laminar termination of ingrowing entorhinal axons in the hippocampus. In addition, the absence of Sema3E/PlexinD1 signalling in postnatal and adult stages also lead to changes in the cytoarchitecture of the dentate gyrus. In their absence, an increased number of stem cell niches are observed in the subgranular zone and newborn granule cells are ectopically settled in the molecular layer. This disorganization strongly alters the laminar distribution and synaptic patterning of mossy fibers. Lastly, these modifications also correlate with the presence of altered functional properties as measured by means of multielectrode local field potential (LFP) recordings, revealing an enhanced excitability in $S e m a 3 E^{0 / 0}$ mice.

\section{Results}

Expression pattern of secreted semaphorins and their receptors in the developing hippocampal formation. Figure 1 shows the low-magnification views of Sema3A, Sema3F, Sema3E, Np1, Np2 and PlxnD1 mRNAs expression in the developing hippocampal formation of E14.5, E16.5 and P0 mice. Due to the anatomical distribution of entorhinal fibers entering the hippocampus ${ }^{5-8}$, we histologically processed horizontal brain sections of the hippocampal formation for in situ hybridization (Fig. 1). Detailed analyses of Sema3A, Sema3F, Np1 and Np2 distribution showed it was similar to that previously reported ${ }^{28}$ although with some differences. Developed sections indicated that both $N p 1$ and $N p 2$ were strongly expressed in the hippocampus between E14.5-E16.5 with decreased levels in the adjacent entorhinal cortex. $N p 1$ labelling, in contrast to $N p 2$, was intense in the entorhinal cortex at E14.5, and absent in the adjacent ventrolateral neocortex between E14.5 and E16.5. In addition, $N p 1$ mRNA labelling was intense in the subiculum compared to $N p 2$ at P0. For ligands, Sema3A staining was relevant in the adjacent ventrolateral neocortex at E14.5-P0, and Sema3F levels in the entorhinal cortex increased from E14.5 to P0. In the hippocampus, Sema3A and 3F displayed similar patterns of labelling from E14.5 to P0 with the pyramidal layer of CA1-3 intensely labelled. Relevantly, the subicular region showed the lowest staining of all three semaphorins in the hippocampal formation at P0 (asterisk in Fig. 1p-r).

PlxnD1 labelling appeared in hippocampal Cajal-Retzius cells at E14.5 (arrow in Fig. 1c), and from E16.5 onwards in the pyramidal cell layer (PCL) of the subiculum-CA1, as previously described ${ }^{32,41}$. In contrast, Sema3E transcript was lightly detected in the emerging dentate gyrus between E14.5-E16.5 and from P0 onwards in the PCL of CA1. In ventrolateral neocortex and entorhinal cortex, PlxnD1 mRNA appeared in upper cortical layers following a lateral to medial gradient of intensity at E14.5. In contrast, Sema3E was present in lower layers of the entorhinal cortex close to the ventricular region from E16.5 (arrows in Fig. 11) to P0. Sense controls displayed no specific signals (Supplementary Fig. 1). In summary, these results indicate that, as reported by Sema3A/3 F and $\mathrm{Np} 1 / 2^{21,28}$, PlexinD1 and Sema3E are expressed in the hippocampal formation during the development of the entorhino-hippocampal connection.

Chemorepulsive action of Sema3E on entorhinal and hippocampal axons during embryonic development. We aimed to explore whether these changes in mRNA expression patterns, especially of $\mathrm{Np} 1$, $N p 2$ and PlxnD1, correlated with particular chemorepulsive actions of secreted semaphorins at E14.5 and E16.5 embryonic stages. These stages correlate with the ingrowth of entorhinal axons in the hippocampus and with the main outgrowth of hippocampal axons (Fig. 1). A summary of the results obtained can be seen in Table 1 and representative examples of explants cultures can be seen in Fig. 2. As indicated, explants showing increased numbers of axons in the distal quadrant in comparison to the proximal (suggesting chemorepulsion) showed a proximal quadrant/distal quadrant $(\mathrm{P} / \mathrm{D})<1$, in contrast to radial outgrowth $(\mathrm{P} / \mathrm{D}=1)$ or chemoattractive 

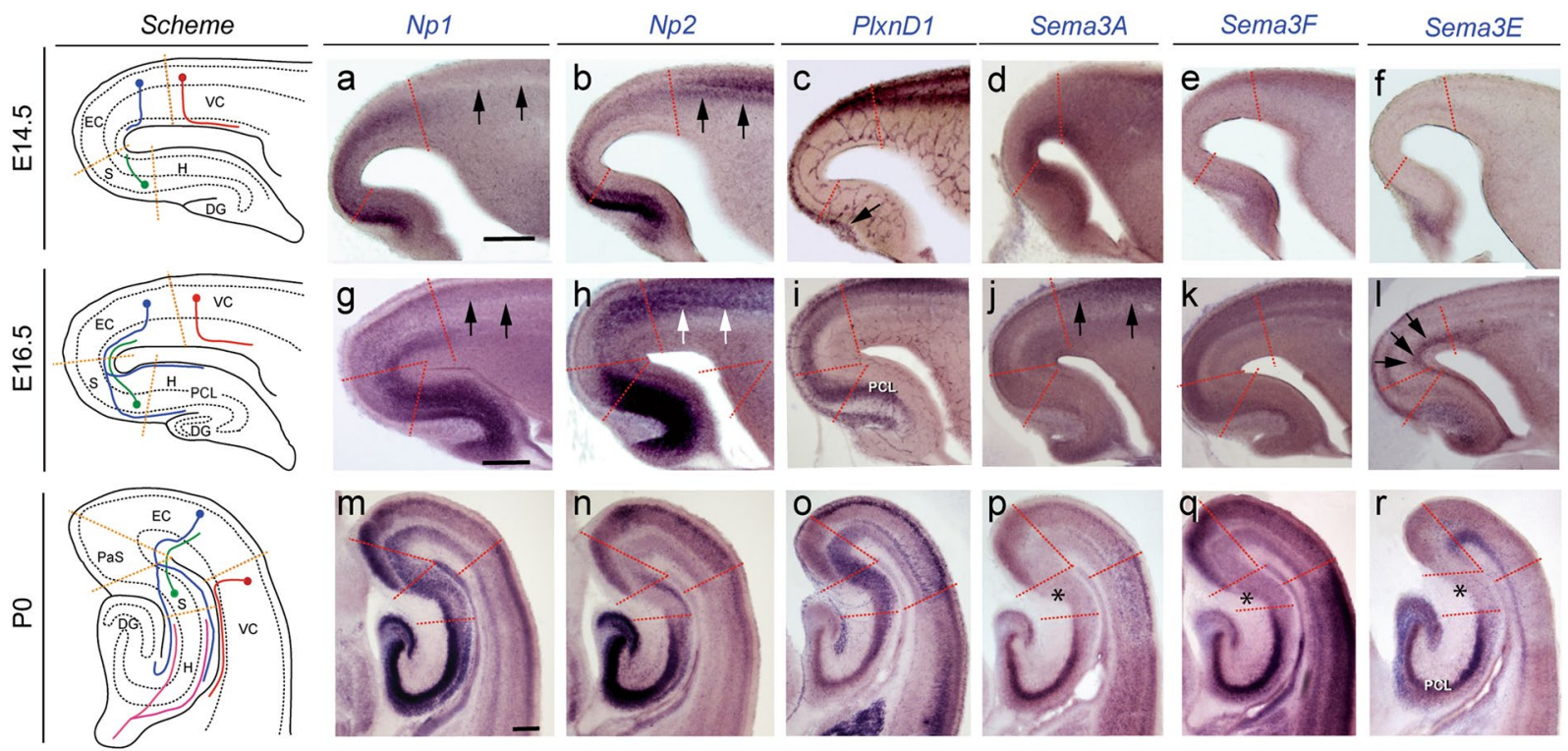

Figure 1. Low-power photomicrographs illustrating the distribution of $N p 1(\mathbf{a}, \mathbf{g}, \mathbf{m}) ; N p 2(\mathbf{b}, \mathbf{h}, \mathbf{n}) ; P \ln x D 1$ $(\mathbf{c}, \mathbf{i}, \mathbf{o}) ; \operatorname{Sema} 3 A(\mathbf{d}, \mathbf{j}, \mathbf{p}) ; \operatorname{Sema} 3 F(\mathbf{e}, \mathbf{k}, \mathbf{q})$ and $\operatorname{Sema} 3 E(\mathbf{f}, \mathbf{l}, \mathbf{r})$ mRNA in the hippocampal formation and adjacent ventrolateral cortex at E14.5 (a-f), E16.5 (g-l) and P0 (m-r). The different regional boundaries are circumscribed by dashed lines. Characteristic corticofugal, entorhino-hippocampal, subiculo-entorhinal and commissural afferent connections are labelled in red, blue, green and pink respectively in the scheme. Note the absence of $N p 1$ labelling in the ventrolateral cortex at E14.5 and E16.5 (arrows in a and g), compared to Np2 (arrows in $\mathbf{b}$ and $\mathbf{h}$ ) and PlnxD1 (c and i). Sema3A levels in the ventrolateral neocortex were intense at E16.5 (arrows in $\mathbf{j}$ ). In addition, Sema3E levels in lower layers of both the ventrolateral and entorhinal cortices can be seen from E16.5 onwards (arrows in 1). Surprisingly, the subicular region was almost absent of semaphorin labelling (asterisk in p-r). Abbreviations: $\mathrm{DG}=$ dentate gyrus; $\mathrm{EC}=$ entorhinal cortex; $\mathrm{H}=$ hippocampus proper; $\mathrm{PaS}=$ parasubiculum; $\mathrm{PCL}=$ pyramidal cell layer; $\mathrm{S}=$ subiculum; $\mathrm{VC}=$ ventrolateral neocortex. Scale bars: $\mathbf{a}=250 \mu \mathrm{m}$ pertains to $(\mathbf{b}-\mathbf{f}) ; \mathbf{g}=250 \mu \mathrm{m}$ pertains to $(\mathbf{h}-\mathbf{l})$ and $\mathbf{m}=100 \mu \mathrm{m}$ pertains to $(\mathbf{n}-\mathbf{r})$.

\begin{tabular}{|c|c|c|c|c|c|c|c|c|c|c|c|c|c|c|c|}
\hline \multirow{2}{*}{$\begin{array}{l}\text { E14.5 } \\
\text { P/D }\end{array}$} & \multicolumn{3}{|l|}{$\mathrm{CA}$} & \multicolumn{3}{|l|}{ SUB } & \multicolumn{3}{|l|}{ EC } & \multicolumn{3}{|l|}{ VC } & \multicolumn{3}{|l|}{ DC } \\
\hline & $<1$ & 1 & $>1$ & $<1$ & 1 & $>1$ & $<1$ & 1 & $>1$ & $<1$ & 1 & $>1$ & $<1$ & 1 & $>1$ \\
\hline S3A & $5(71.4 \%)$ & $1(14.3 \%)$ & $1(14.3 \%)$ & $2(18.18 \%)$ & $2(18.18 \%)$ & $7(63.63 \%)$ & $3(23.08 \%)$ & $4(30.77 \%)$ & $6(46.15 \%)$ & $4(30.77 \%)$ & $8(61.54 \%)$ & $1(7.69 \%)$ & $4(25 \%)$ & $9(56.25 \%)$ & $3(18.75 \%)$ \\
\hline S3F & $6(54.54 \%)$ & $2(18.18 \%)$ & $3(27.27 \%)$ & $2(14.28 \%)$ & $8(57.14 \%)$ & $4(28.57 \%)$ & $3(21.43 \%)$ & $7(50 \%)$ & $4(28.57 \%)$ & $7(63.63 \%)$ & $3(27.27 \%)$ & $1(9.1 \%)$ & $6(40 \%)$ & $6(40 \%)$ & $3(20 \%)$ \\
\hline S3E & $14(87.5 \%)$ & $2(12.5 \%)$ & $0(0 \%)$ & $12(75 \%)$ & $4(25 \%)$ & $0(0 \%)$ & \begin{tabular}{|l|}
15 \\
$(93.75 \%)$
\end{tabular} & $1(6.25 \%)$ & $0(0 \%)$ & $12(85.71 \%)$ & $2(14.29 \%)$ & $0(0 \%)$ & $0(0 \%)$ & $10(90.9 \%)$ & $1(9.1 \%)$ \\
\hline SEAP & $4(66.66 \%)$ & $2(33.33 \%)$ & $0(0 \%)$ & $5(71.43 \%)$ & $2(28.57 \%)$ & $0(0 \%)$ & $2(66.66 \%)$ & $1(33.33 \%)$ & $0(0 \%)$ & $2(66.66 \%)$ & $1(33.33 \%)$ & $0(0 \%)$ & $3(37.5 \%)$ & $5(62.5 \%)$ & $0(0 \%)$ \\
\hline E16.5 & \multicolumn{3}{|l|}{ CA } & \multicolumn{3}{|l|}{ SUB } & \multicolumn{3}{|l|}{ EC } & \multicolumn{3}{|l|}{ VC } & \multicolumn{3}{|l|}{ DC } \\
\hline $\mathrm{P} / \mathrm{D}$ & $<1$ & 1 & $>1$ & $<1$ & 1 & $>1$ & $<1$ & 1 & $>1$ & $<1$ & 1 & $>1$ & $<1$ & 1 & $>1$ \\
\hline $\mathrm{S} 3 \mathrm{~A}$ & $6(42.85 \%)$ & $6(42.85 \%)$ & $2(14.3 \%)$ & \begin{tabular}{|l|}
10 \\
$(66.66 \%)$
\end{tabular} & $5(33.33 \%)$ & $0(0 \%)$ & $5(41.66 \%)$ & $6(50 \%)$ & $1(8.33 \%)$ & $4(33.33 \%)$ & $6(50 \%)$ & $2(16.67 \%)$ & $3(23.08 \%)$ & $7(53.84 \%)$ & $3(23.08 \%)$ \\
\hline S3F & $11(100 \%)$ & $0(0 \%)$ & $0(0 \%)$ & $9(60 \%)$ & $5(33.33 \%)$ & $1(6.67 \%)$ & $14(77.7 \%)$ & $3(16.67 \%)$ & $1(5.56 \%)$ & $14(100 \%)$ & $0(0 \%)$ & $0(0 \%)$ & $2(18.18 \%)$ & $6(54.54 \%)$ & $3(27.27 \%)$ \\
\hline S3E & $12(75 \%)$ & $4(25 \%)$ & $0(0 \%)$ & $9(60 \%)$ & $6(40 \%)$ & $0(0 \%)$ & $9(40.9 \%)$ & $11(50 \%)$ & $2(9.1 \%)$ & $9(56.25 \%)$ & $7(43.75 \%)$ & $0(0 \%)$ & $4(33.33 \%)$ & $3(25 \%)$ & $5(41.67 \%)$ \\
\hline SEAP & $3(30 \%)$ & $7(70 \%)$ & $0(0 \%)$ & $5(50 \%)$ & $4(40 \%)$ & $1(10 \%)$ & $1(10 \%)$ & $7(70 \%)$ & $2(20 \%)$ & $3(33.33 \%)$ & $4(44.44 \%)$ & $2(22.23 \%)$ & $3(42.86 \%)$ & $4(57.14 \%)$ & $0(0 \%)$ \\
\hline
\end{tabular}

Table 1. Effects of Sema3A, Sema3F and Sema3E on the growth of CA1-3, entorhinal, subicular, ventrolateral and dorsal cortical axons in confrontation experiments (see Material and methods for details). The effects of these class III secreted semaphorins on the experiments are summarized as $\mathrm{P} / \mathrm{D}<1, \mathrm{P} / \mathrm{D}=1$ or $\mathrm{P} / \mathrm{D}>1$, indicating chemorepulsion, radial outgrowth or chemoattraction, respectively. For each region the number of cultures displaying these effects is shown. Abbreviations: CA =CA1-3 hippocampal regions; $\mathrm{D}=$ distal quadrant $\mathrm{DC}=$ dorsal cortex $\mathrm{EC}=$ entorhinal cortex $\mathrm{P}=$ proximal quadrant; $\mathrm{SUB}=$ subiculum; $\mathrm{VC}=$ ventrolateral cortex.

$(\mathrm{P} / \mathrm{D}>1)$ responses (see ${ }^{46}$ and Supplementary Fig. 2a for details). Results indicated that Sema3E was able to induce chemorepulsion of CA1-3 (E14.5; $\approx 87.5 \%$; $\mathrm{P} / \mathrm{D}$ ratio $<1 ; \mathrm{n}=16)$, subiculum $(\mathrm{E} 14.5 ; \mathrm{P} / \mathrm{D}$ ratio $<1$; $\approx 75 \% ; \mathrm{n}=16)$, entorhinal cortex $(\mathrm{E} 14.5 ; \mathrm{P} / \mathrm{D}$ ratio $<1 ; \approx 93.75 \% ; \mathrm{n}=16)$, and ventrolateral $(\mathrm{E} 14.5 ; \mathrm{P} / \mathrm{D}$ ratio $<1$; $\approx 85.71 \% ; \mathrm{n}=14$ ) but not dorsal (parietal) neocortex axons at E14.5. $(\mathrm{E} 14.5 ; \approx 90.9 \% ; \mathrm{P} / \mathrm{D}$ ratio $=1 ; \mathrm{n}=11)$. At later embryonic stages (E16.5) these chemorepulsive actions were largely maintained ((CA1-3; $\approx 75 \% ; P / D$ 

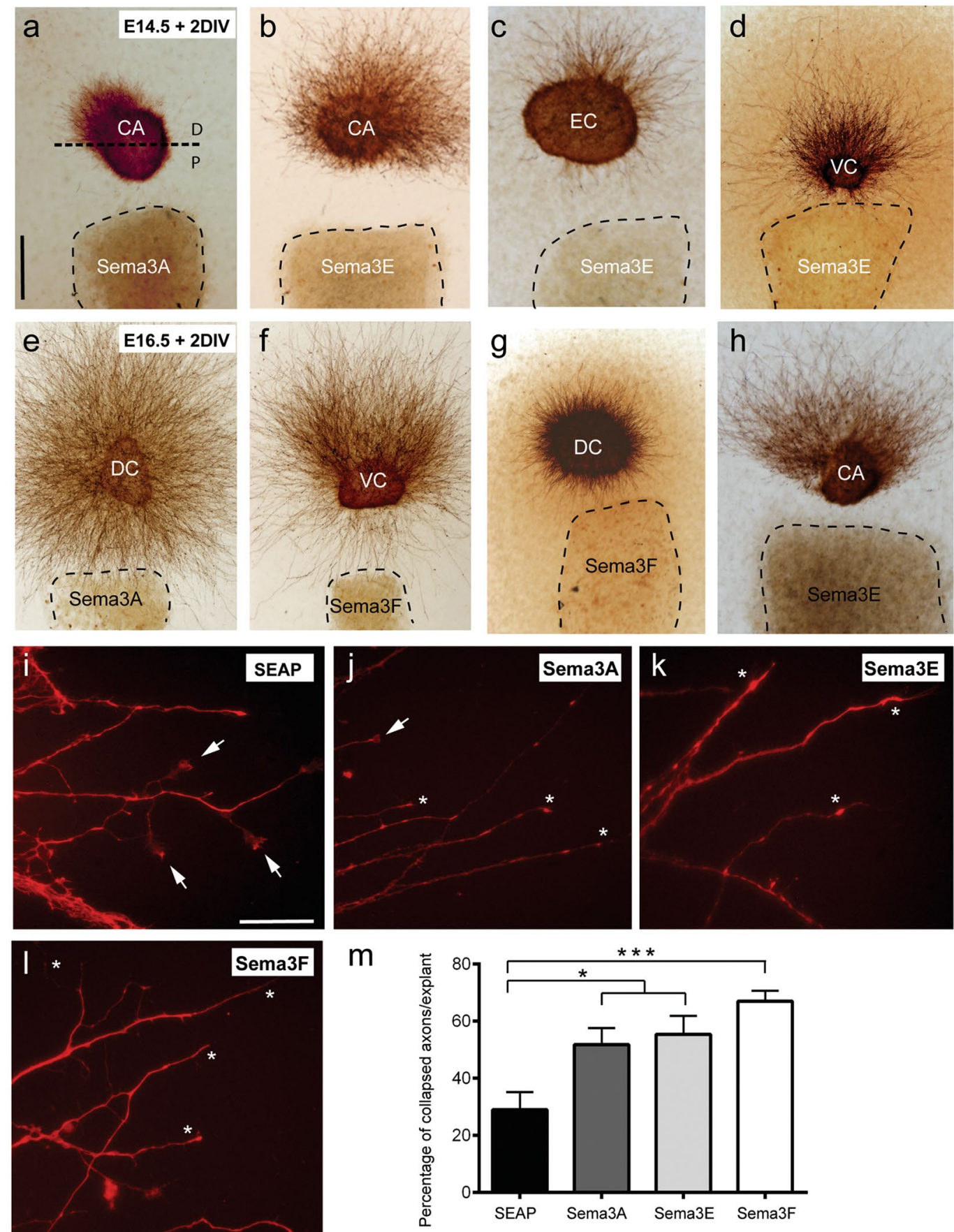

Figure 2. Low-power photomicrographs showing examples of the chemorepulsion of hippocampal (a,b,h), entorhinal (c), ventrolateral (d and $\mathbf{f}$ ) and dorsal (e and $\mathbf{g}$ ) axons by Sema3A, Sema3F and Sema3E. Explants were obtained at E14.5 (a-d) or E16.5 (e-h), cultured for 2 days in vitro (DIV) and processed for $\beta$ III-tubulin (clone TUJ-1) immunostaining. (a) Dotted line defines the boundary between the proximal (P) and the distal (D) quadrant of the explants. Note the strong chemorepulsion of hippocampal axons by Sema3A at E14.5. In addition, Sema3E-mediated chemorepulsion can be seen on hippocampal, entorhinal and ventrolateral cortex at E14.5 (b-d). Examples of Sema3F-mediated chemorepulsion on ventrolateral axons and Sema3Eeffects on hippocampal axons can be seen in $\mathbf{f}$ and $\mathbf{h}$ respectively. This contrasts with what is observed for dorsal neocortical axons $(\mathbf{g})$. (i-m) Representative phalloidin-TRITC stained neuronal processes of cultured entorhinal explants from E16.5, illustrating semaphorin-mediated growth cone collapse. (i) Normal growth cones, with lamellipodia and filopodia (arrows) from entorhinal explants cultured with SEAP medium. (j-l) Examples of collapsed growth cones (asterisks) after incubation with Sema3A (j), Sema3E (k) and Sema3F (l). (m) Histogram illustrating percentages of collapsed growth cones per explant after the incubation of entorhinal explants with secreted semaphorins. Results represent the mean \pm S.E.M. of three separate experiments. Asterisks indicate statistical differences between groups and controls. $* * * P \leq 0.001$; ANOVA Bonferroni post hoc test. Abbreviations: $\mathrm{CA}=\mathrm{CA1}-3$ hippocampal regions; $\mathrm{EC}=$ entorhinal cortex; $\mathrm{DC}=$ dorsal neocortex; $\mathrm{VC}=$ ventrolateral neocortex. Scale bars: $\mathbf{a}=150 \mu \mathrm{m}$ pertains to $(\mathbf{b}-\mathbf{h}) ; \mathbf{i}=20 \mu \mathrm{m}$ pertains to $(\mathbf{j}-\mathbf{l})$. 
ratio $<1 ; \mathrm{n}=16$ ); (subiculum; $\approx 60 \% ; \mathrm{P} / \mathrm{D}$ ratio $<1 ; \mathrm{n}=15$ ) (ventrolateral neocortex; $\approx 56.25 \%$; $\mathrm{P} / \mathrm{D}$ ratio $<1$; $\mathrm{n}=16)$ ) except for entorhinal cortex $(\mathrm{E} 16.5 ; \approx 41 \% ; \mathrm{P} / \mathrm{D}$ ratio $=1 ; \mathrm{n}=22)$. These effects largely correlate with PlxnD1 mRNA distribution (Fig. 1). Sema3E-induced chemorepulsive effect on entorhinal axons at E14.5 was analysed in explants derived from Nestin-cre; PlxnD1 flox/flox mice (Supplementary Fig. 2d). In this case, we observed that Nestin-cre; PlxnD $1^{\text {flox/flox }}$ E14.5 entorhinal explants showed a drastic reduction in chemorepulsive response $(\approx 54.5 \% ; \mathrm{P} / \mathrm{D}$ ratio $<1 ; \approx 45.5 \% ; \mathrm{P} / \mathrm{D}$ ratio $=1 ; \mathrm{n}=22)$. In addition, Sema3A elicited clear chemorepulsive action on CA1-3 axons at $\mathrm{E} 14.5(\approx 71.4 \% ; \mathrm{P} / \mathrm{D}$ ratio $<1 ; \mathrm{n}=7)$ with decreasing effects at $\mathrm{E} 16.5(\approx 42.8 \% ; \mathrm{P} / \mathrm{D}$ ratio $<1 ; \mathrm{n}=14$ and $\approx 42.8 \% ; \mathrm{P} / \mathrm{D}$ ratio $=1 ; \mathrm{n}=14)$. In contrast, Sema3F-mediated chemorepulsion on CA1-3 axons increased from $\mathrm{E} 14.5(\approx 54.5 \% ; \mathrm{P} / \mathrm{D}$ ratio $<1 ; \mathrm{n}=6)$ to $\mathrm{E} 16.5(\approx 100 \% ; \mathrm{P} / \mathrm{D}$ ratio $<1 ; \mathrm{n}=11)$. For ventrolateral neocortex, Sema3F-mediated chemorepulsion was observed at E14.5 $(\approx 63.6 \%$; $/ \mathrm{D}$ ratio $<1 ; \mathrm{n}=11)$ but relevantly at $\mathrm{E} 16.5(\approx 100 \% ; \mathrm{P} / \mathrm{D}$ ratio $<1 ; \mathrm{n}=14)$. As indicated above, we were unable to determine any chemorepulsive effect in dorsal neocortex at these stages (Fig. 2g; Table 1). With respect to entorhinal axons we observed that chemorepulsion mainly took place at E16.5 for Sema3F $(\approx 77.7 \%$; $/ D$ ratio $<1 ; \mathrm{n}=18)$ and with a tendency for Sema3E $(\approx 41 \% ; \mathrm{P} / \mathrm{D}$ ratio $<1$ and $\approx 50 \% ; \mathrm{P} / \mathrm{D}$ ratio $=1 ; \mathrm{n}=22)$ and Sema3A $(\approx 41.6 \% ; \mathrm{P} / \mathrm{D}$ ratio $<1$ and $\approx 50 \% ; \mathrm{P} / \mathrm{D}$ ratio $=1 ; \mathrm{n}=12$ ). These last results were corroborated in collapse experiments on identified growth cones of entorhinal cortex explants at E16.5 (Fig. 2i-m). All three semaphorins induced collapse of growth cones with the strongest effects induced by Sema3F (Fig. 2l,m) (e.g., Sema3F; 66.94 \pm 3.68; vs SEAP; $28.95 \pm 6.210$; Mean \pm S.E.M.; $t=4.954 ; P=0.0005$; confidence interval $95 \%=-60.57$ to -15.42 ; ANOVA, Bonferroni post hoc test).

Altered pattern of the EH connection in absence of PlexinD1 and Sema3E. Since PlxnD1/0 mice die prenatally, we crossed $P l x n D 1^{\text {flox } f l o x}$ with Nestin-cre mice. In the resulting conditional mutant Nestin-cre; PlxnD $1^{\text {flox/flox }}$, the decrease of PlxnD1 mRNA was corroborated histologically by in situ hybridization at P0 (Supplementary Fig. 3b). As Nestin promoter is active from E12-E13, early-generated hippocampal Cajal-Retzius cells were labelled by PlxnD1 probes at P0. In addition, blood vessels were also labelled with PlxnD1 probes in some brain regions (Supplementary Fig. 3b). Absence of PlxnD1 did not alter Sema3E, Np1 or Np2 (Supplementary Fig. 3c-f) or PlxnA1 expression (not shown).

Organotypic slices of the hippocampal formation have been used largely to determine the development and layer specific targeting of entorhinal ${ }^{13,47}$ septal $^{29}$ and commissural connections ${ }^{19}$ in several experimental conditions. Thus, taking advantage of this culture preparation, we established EH organotypic slice cultures from newborn mutant Nestin-cre; PlxnD $1^{\text {flox/flox }}$ mice (Supplementary Fig. 3g,h). After byocitin tracing, the entorhino-hippocampal connection was formed and entorhinal axons were able to cross the subiculum, reaching the hippocampal slm/ml in Nestin-cre; PlxnD flox/+ and Nestin-cre; PlxnD1 $1^{\text {flox/flox }}$ entorhino-hippocampal cocultures (Supplementary Fig. 3g,h). However, numerous byocitin-positive misrouted fibers were seen in the CA1 and in the DG (red asterisks in Supplementary Fig. 3h) of Nestin-cre; PlxnD1 flox/flox cultures compared to their control littermate Nestin-cre; PlxnD flox/+ (Supplementary Fig. 3g). Next we traced the EH connection with the anterograde lipophilic tracer DiI at P0 in Sema3 $E^{0 / 0}$ and control mice (Fig. 3). In control (wild-type and Sema3 $E^{+/ 0}$ ) mice, DiI axons followed the perforant pathway, being restricted to the slm (arrows in Fig. 3a) and the white matter. In contrast, in Sema3 $E^{0 / 0}$ mice (Fig. 3b-f) the EH connection was formed, but numerous axons crossed the hippocampal fissure, perforating the granule cell layer and reaching the dentate hilus (arrowheads in Fig. 3c-f). In addition, large numbers of DiI-labeled ectopic axons were also observed crossing the subiculum-CA1 from the hippocampal alveolar path towards the slm (arrowheads in Fig. $3 \mathrm{c}-\mathrm{f}$ ). These effects were seen in all mutant mice $(n=10)$ from three different litters of Sema $3 E^{0 / 0}$ mice. In other words, the absence of Sema3E or PlexinD1 led to misrouted entorhinal axons during the establishment of the entorhino-hippocampal connection.

Increased proliferation and ectopic granule cells in absence of Sema3E or PlxnD1. To further investigate whether absence of Sema3E/PlexinD1 signalling could lead to other abnormalities, we processed brain sections from different postnatal (P9 and P15) and adult stages to check the cytoarchitecture of the hippocampal formation (Figs 4 and 5) and putative changes in axonal connections (Fig. 6). In histological analysis from P9 onwards, hippocampus from Sema3 $E^{0 / 0}$ showed an unshaped dentate gyrus with the presence of several waves of the suprapyramidal blade of dentate gyrus (arrowheads in Fig. 4a-c) and with a great

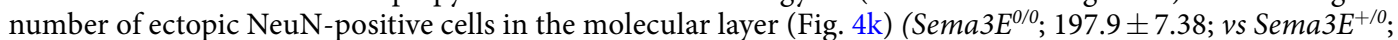
$108.7 \pm 5.49$; Mean \pm S.E.M.; $t=9.699 ; P<0.0001$; confidence interval $99 \%=63.43$ to 115.1 ; unpaired $t$ test with Welch's correction, $* * * * P \leq 0.0001)$. These changes were also seen, although less pronounced, in adult Nestin-cre; $P l x n D 1^{f l o x / f l o x}$ mice (Fig. 4f). Presence of misallocated granule cells was validated with different staining such as double immunofluorescence for Calretinin-Calbindin (Fig. 4d,g,i) or single immunostaining of Prox-1(Fig. 4e). Double-labelled (Calretinin-Calbindin) sections illustrated the presence of ectopic Calbindin-positive cells outside the granule cell layer crossing the inner molecular layer (iml) of the suprapyramidal blade in $\operatorname{Sema} 3 \mathrm{E}^{0 / 0}$ mice at all stages analysed (Fig. 4d,i). Next, we aimed to determine the cause of the abnormal distribution of granule cells in the hippocampus. To this end, we intraperitoneally injected adult mice with BrdU for 4 days and, one week later, mice were processed for immunohistochemistry. Cell counts increased, although this was not statistically significant, for the numbers of BrdU-positive cells in the granule cell layer of Sema3 $E^{0 / 0}$ mice (Fig. 4l). BrdU-immunoreacted sections also showed numerous BrdU-positive cells at different levels of the granule cell layer, often forming columns spanning the layer in $S e m a 3 E^{0 / 0}$ mice (arrows in Fig. 4j). Indeed, we detected an increase in BrdU-positive cells in the outer portion of the granule cell layer of Sema3 $E^{0 / 0}$ compared to Sema3 $E^{+/ 0}$ and Sema3 $E^{+/+}$mice (Fig. 4h,j,m) $\left(\right.$Sema3 $E^{0 / 0} ; 5.67 \pm 1.11$; vs Sema3 $E^{+/ 0} ; 3.0 \pm 0.61$; Mean \pm S.E.M.; $t=2.291$; $P=0.0849$; confidence interval $90 \%=-5.248$ to $-0.084 ;$ Sema3 $E^{0 / 0} ; 5.67 \pm 1.11 ;$ vs Sema3 $E^{+/+}$mice; $3.14 \pm 0.57$; $t=2.301 ; P=0.083$; confidence interval $90 \%=-4.957$ to -0.09061 ; ANOVA, Bonferroni post hoc test, $* P<0.1)$. The presence of different immature cells in different locations of the granule cell layer was also observed with 

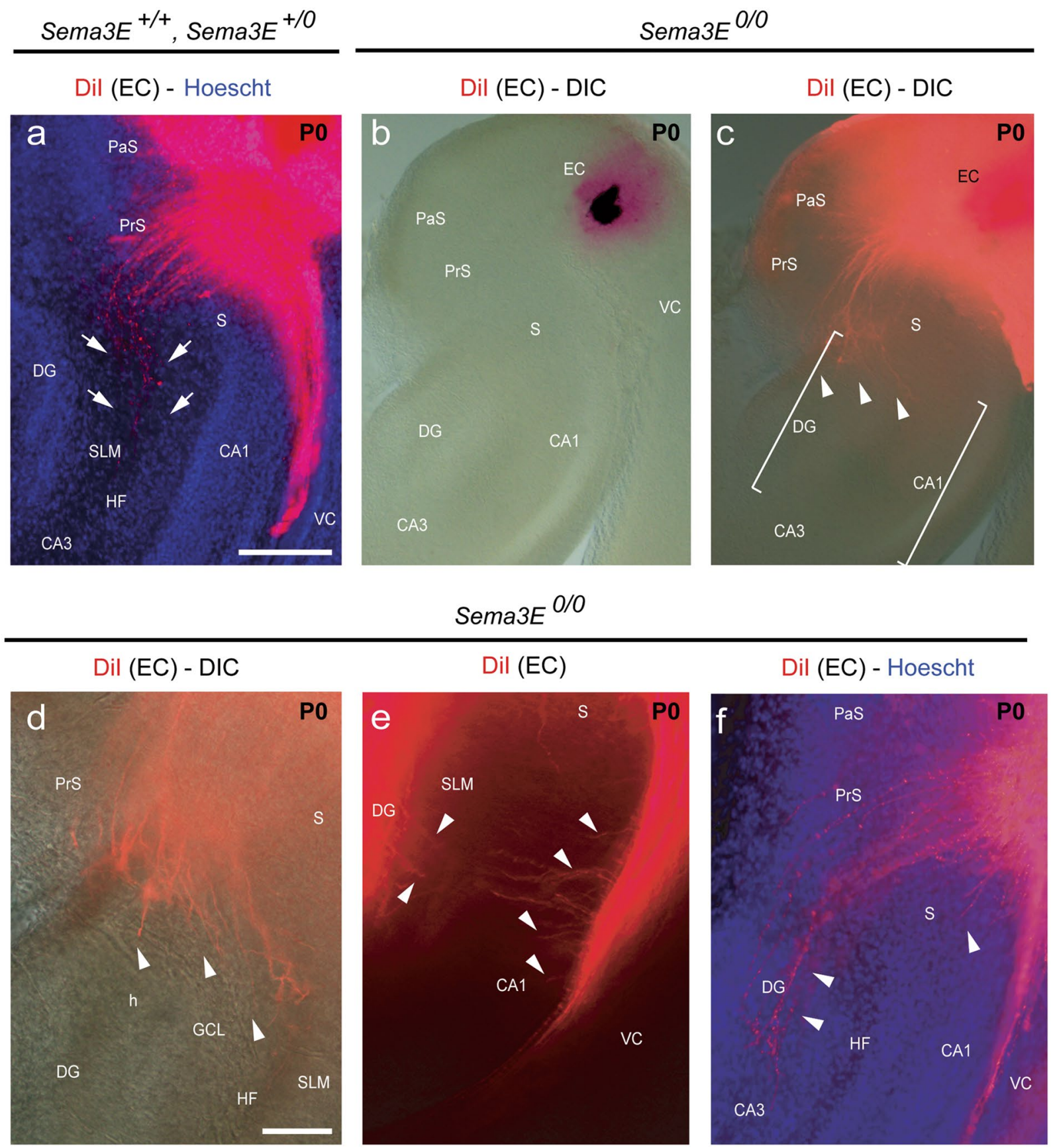

Figure 3. Pattern of entorhino-hippocampal innervations in Sema3E-deficient mice after DiI injections in the entorhinal cortex at P0. (a) In wild-type and Sema3 $E^{+/ 0}$ mice, entorhinal fibers are restricted to the stratum lacunosum-moleculare (arrows) and the white matter. (b-f) In Sema3 $E^{0 / 0}$ mice the EH connection is formed, but ectopic axons cross the hippocampal fissure, entering the dentate gyrus or the CA1 (arrowheads in b-f). Abbreviations as in Fig. 1 and $\mathrm{GCL}=$ granule cell layer; $\mathrm{h}=$ hilus; $\mathrm{HF}=$ hippocampal fissure; $\mathrm{PrS}=$ presubiculum; $\mathrm{SLM}=$ stratum lacunosum- moleculare. Scale bar: $\mathbf{a}=250 \mu \mathrm{m}$ pertains to $(\mathbf{b}-\mathbf{c})$; $\mathbf{d}$ pertains to $(\mathbf{e}-\mathbf{f})=100 \mu \mathrm{m}$.

electron microscopy techniques (Supplementary Fig. 4). In these sections, both ectopic granule cells in the molecular layer and immature cells located in cell niches of the subgranular layer were clearly observed (Supplementary Fig. $4 a, b)$. Sema $3 E^{0 / 0}$ mutant mice exhibited a larger number of cell niches with more immature cells within them. As a consequence, the subgranular contour of these animals is more irregular when compared to the same region in Sema3 $E^{+/ 0}$ animals (Supplementary Fig. $4 \mathrm{a}-\mathrm{d}$ ). In parallel, due to the relevance of the vascular system in stem cell niche biology we aimed to determine changes in vasculature in the hippocampus of $S e m a 3 E^{0 / 0}$ mice. However, the analysis of blood vessel distribution in the hippocampus revealed no relevant alterations between control and mutant mice (Supplementary Fig. 5). In conclusion, absence of Sema3E led to increased proliferation of the subgranular zone precursors of the adult dentate gyrus. 


\section{Sema3E $0 / 0$}
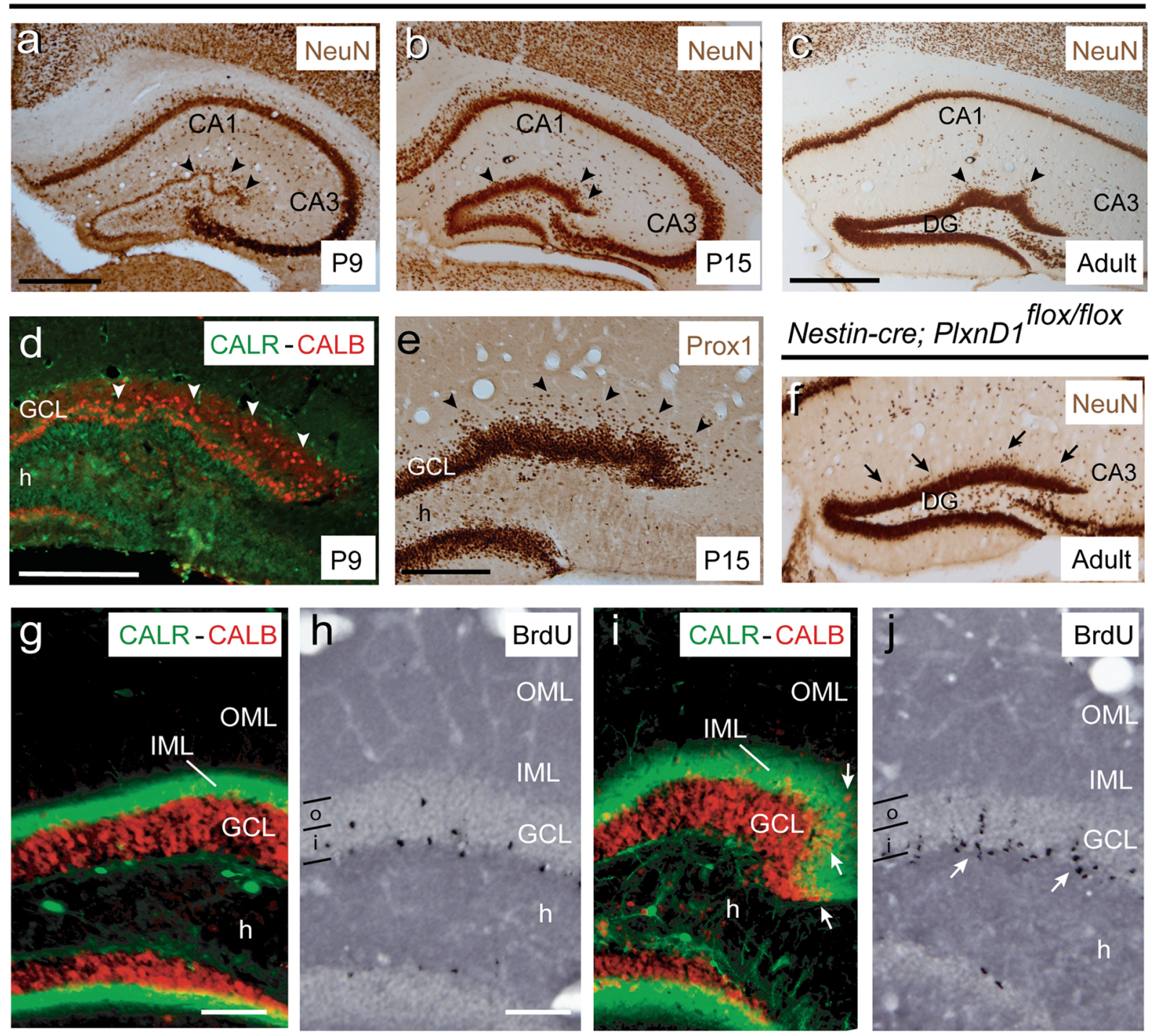

k

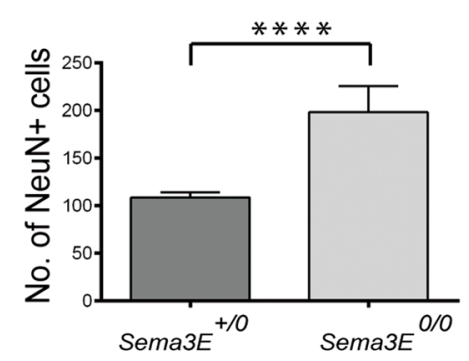

I

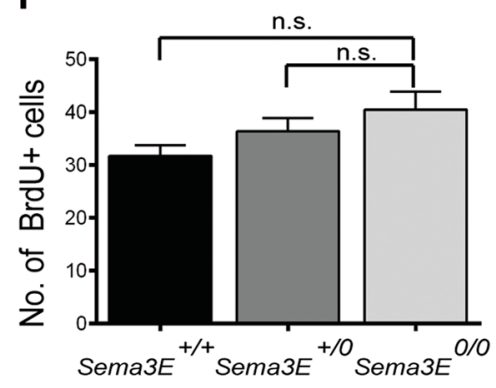

$\mathrm{m}$

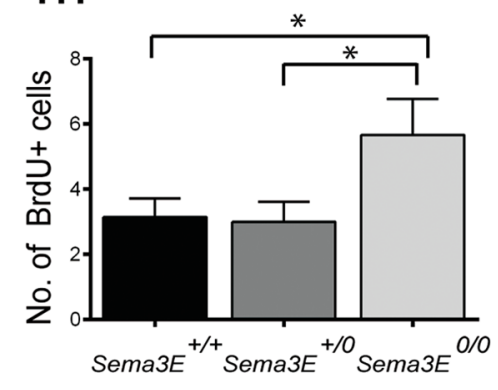

Figure 4. Examples of $\alpha-\mathrm{NeuN}$ immunostaining in the hippocampus proper and dentate gyrus of $\operatorname{Sema3} E^{0 / 0}$ $(\mathbf{a}-\mathbf{c})$ and Nestin-cre; PlxnD flox/flox $(\mathbf{f})$ mice at different postnatal stages. Note the presence of numerous NeuNpositive cells in the molecular layer in absence of Sema3E/PlexinD1 signalling (arrowheads), especially in Sema3E-deficient mice. Also note the presence of several waves of the suprapyramidal blade of dentate gyrus. (d) Double immunolabeling of Calretinin (green) and Calbindin (red) in the dentate gyrus of Sema3 $E^{0 / 0}$ mice at P9. Note the presence of numerous ectopic Calbindin-positive neurons in the IML of Sema3E $E^{0 / 0}$ mice (arrowheads). (e) Prox-1 immunolabeling in the dentate gyrus of Sema3E $E^{0 / 0}$ mice at P15 shows the presence of numerous Prox-1-positive granule cells in the molecular layer (arrowheads). (g,i) Double immunolabeling of Calretinin (green) and Calbindin (red) in the dentate gyrus of control (g) and Sema3 $E^{0 / 0}$ adult mice (i). Note the presence of numerous ectopic Calbindin-positive neurons in the IML of Sema3 $E^{0 / 0}$ mice (arrows in $\mathbf{i})$. (h-j) Examples of BrdU-labeled neurons in the granule cell layer of control (h) and $\operatorname{Sema} 3 E^{0 / 0}(\mathbf{j})$ mice. Numerous BrdU-positive cells forming columns in the granule cell layer can be seen in mutant mice (arrows 
in j). (k) Histogram illustrating the number of NeuN-positive cells in the IML of the suprapyramidal blade of the dentate gyrus in Sema3 $E^{+/ 0}$ and Sema3 $E^{0 / 0}$ mice. (1-m) Histograms illustrating the number of BrdUpositive cells counted in the whole granule cell layer (1) and in the outer portion of the granule cell layer of the dentate gyrus (labeled as 'o') (m) in Sema3 $E^{+/+}$, Sema3 $E^{+/ 0}$ and Sema3 $E^{0 / 0}$ mice. Asterisks indicate statistical differences between groups. $* P \leq 0.05 ; * * P \leq 0.01 ; * * * P \leq 0.001$; $* * * * P \leq 0.0001$. ANOVA Bonferroni post hoc test. Abbreviations as in Figs $1-3$ and $\mathrm{i}=$ inner portion of the granule cell layer; $\mathrm{IML}=$ inner molecular layer; $\mathrm{o}=$ outer portion of the granule cell layer and $\mathrm{OML}=$ outer molecular layer. Scale bars: $\mathbf{a}=\mathbf{b}=500 \mu \mathrm{m}$; $\mathbf{c}=\mathbf{f}=500 \mu \mathrm{m} ; \mathbf{d}=200 \mu \mathrm{m} ; \mathbf{e}=200 \mu \mathrm{m} ; \mathbf{g}$ and $\mathbf{h}=150 \mu \mathrm{m}$ pertains to $\mathbf{i}$ and $\mathbf{j}$ respectively.

PlexinD1 is expressed in type-1 radial glia-like stem cells. After the description of the dysregulation of cell proliferation in subgranular cells, we next explored whether Sema3E/PlexinD1 signalling could influence adult neurogenesis. First, we determined PlexinD1 expression in the adult hippocampus of PlxnD1-eGFP mice using GFP antibody and we observed cellular PlexinD1 labelling in subsets of pyramidal CA1neurons and in cells from the hilus and subgranular zone (Fig. $5 \mathrm{a}-\mathrm{c}$ ) suggesting that it could be expressed by proliferating cells. The presence of PlexinD1-expressing cells in the dentate gyrus has also been reported in ${ }^{48}$ and published on the Allen Brain Atlas web site (http://mouse.brain-map.org, experiment 73521005). Additionally, Sema3E mRNA expression was detected in the pyramidal layer and the stratum lacunosum-moleculare of CA1-3 and in the hilus with lesser presence in the granule cell layer (Fig. $5 \mathrm{~d}, \mathrm{e}$ ). To identify cell types expressing GFP in the subgranule zone of PlxnD1-eGFP mice we performed double immunofluorescence with different neurogenic markers and GFP. Double-labelled (GFP/GFAP) sections illustrated that GFP was present in numerous type-1 radial GFAP-positive cells in the subgranular zone (Fig. 5f-i) but absent in immature neurons (DCX-positive neurons) (Fig. 5j-l), leading us to conclude that PlexinD1 is present in radial type-1 glia-like stem cells in the subgranular zone.

Aberrant mossy fiber distribution in absence of Sema3E/PlexinD1 signalling. Next we analysed the distribution of the hippocampal connections in adult mice lacking Sema3E/PlexinD1 signalling (Fig. 6). BDA labelling of EH axons in adult Sema $3 E^{0 / 0}$ mice revealed no relevant changes in the distribution of labelled entorhinal axons in the CA1-3 regions and layer $(\mathrm{slm} / \mathrm{ml})$ or in the wavy distribution of the $\mathrm{EH}$ axons in the dentate molecular layer (data not shown). In parallel, sections of Sema3E-deficient mice immunostained with $\alpha$-Calretinin antibody (to label commissural-associational connections in the dentate molecular layer) showed diffuse puncta-like labelling in the inner portion of the dentate molecular layer compared to Sema3 $E^{+/+}$and Sema $3 E^{+/ 0}$ mice (Fig. 6a,b). Calretinin-positive labelling in the iml was apposed to the wavy granule cell layer (Fig. 6b). Parallel sections processed for Calbindin immunostaining showed that the coarse distribution of the mossy fiber projections (supra and infrapyramidal) was similar in mutant and control mice (not shown).

However, after selenite-silver staining for zinc-rich projections we observed profound changes in the mossy fiber distribution in the dentate gyrus of the Sema $3 E^{0 / 0}$ mice with numerous ectopic fibers in the iml, especially in the gyri of the waves reaching the oml (Fig. 6c-f). Mossy fiber sprouting was further corroborated in parallel sections immunoprocessed for the detection of the presynaptic marker Synaptoporin (SPO) (Fig. 6g,h). SPO-immunoreacted sections revealed that some mossy fibers sprouted into the granule cell layer of Sema3E-deficient mice (Fig. 6h). This aberrant mossy fiber distribution was observed in all the mutant animals analysed from four different $S e m a 3 E^{0 / 0}$ litters.

Finally, we analysed sections from Nestin-cre; PlxnD $1^{f l o x}$ flox mice in order to detect changes in the mossy fiber distribution. As observed in the absence of Sema3E, mice lacking PlexinD1 (Nestin-cre; PlxnD1 $1^{\text {flox/flox }}$ ) also showed aberrant sprouting into the granule cell layer compared to their control littermates (Nestin-cre; PlxnD $1^{\text {flox/+ }}$ ) (Fig. 6i,j). In conclusion, mice lacking either Sema3E or PlexinD1 showed relevant changes in the layer patterning of hippocampal mossy fibers.

Dysregulation of excitability in Sema3 $E^{0 / 0}$ hippocampus. The presence of aberrant synaptic connections in the dentate gyrus has been described in several models of epilepsy ${ }^{49,50}$. The unveiling of ectopic mossy fiber terminals in the dentate gyrus of $S e m a 3 E^{0 / 0}$ mice opened the question of whether the animals would functionally show epileptic-like activity patterns or enhanced neural excitability. To answer this, we recorded spontaneous local field potential (LFP) activity by means of a 16-channel multi-electrode array (100 $\mu \mathrm{m}$ spacing) covering the DG and CA1 areas of the hippocampus and the overlying neocortex (Fig. 7a,b) in a cohort of 8 mice ( 4 Sema3 $E^{0 / 0}$ and 4 Sema $3 E^{+/+}$) under deep anesthesia (see Materials and methods for details). Under these conditions, the brain activity is dominated by the characteristic dynamics of the slow-wave oscillatory activity (Fig. 7c), consisting of periods of high-firing activity called Up states, interspersed with almost silent epochs known as Down states ${ }^{51-53}$. Careful observation of the LFP profile revealed no evidence of epileptiform activity patterns in either group of animals (data not shown). Furthermore, in a subset of $3 \mathrm{Sema} 3 E^{+/+}$and $3 \mathrm{Sema}^{2} E^{0 / 0}$ animals, administration of the epileptogenic kainic acid $(10 \mathrm{mg} / \mathrm{kg}$, ip) revealed no differences in the dynamics of epileptic epochs between genotypes. Neither the latency to first discharge (Sema3E $E^{0 / 0}: 2400 \pm 400 \mathrm{~s}, \operatorname{Sema3E}^{+/+}$: $1800 \pm 600 \mathrm{~s})$ nor the frequency of epileptic epochs $\left(\operatorname{Sema}^{0 / 0}: 0.03 \pm 0.02 \mathrm{~Hz}, \operatorname{Sema}^{0} E^{+/+}: 0.02 \pm 0.01 \mathrm{~Hz}\right)$ or their duration $\left(\operatorname{Sema3}^{0 / 0}: 27 \pm 3 \mathrm{~s}\right.$, Sema3 $E^{+/}: 31 \pm 11 \mathrm{~s}$ ) were significantly different between $\operatorname{Sema} 3 E^{0 / 0}$ and Sema3 $E^{+/+}$animals ( $\mathrm{P}>0.05$, Wilcoxon rank-sum test for all comparisons). Therefore, animals lacking Sema3E not only lacked spontaneous epileptic discharges, but they also lacked an enhanced tendency to express epileptiform activity patterns. Next, the spontaneous physiological activity, characterized by a slow oscillatory signal, was quantified by measuring parameters of the Up and Down states as reported in other mouse models ${ }^{52}$. Analysis of the multiunit activity revealed a trend towards the elongation of Up states in the CA1 and dentate gyrus of Sema3 $E^{0 / 0}$ (Fig. 7c,d, green) with respect to Sema3 $E^{+/+}$(Fig. 7c,d, black) animals $(P<0.1$, Wilcoxon rank-sum 


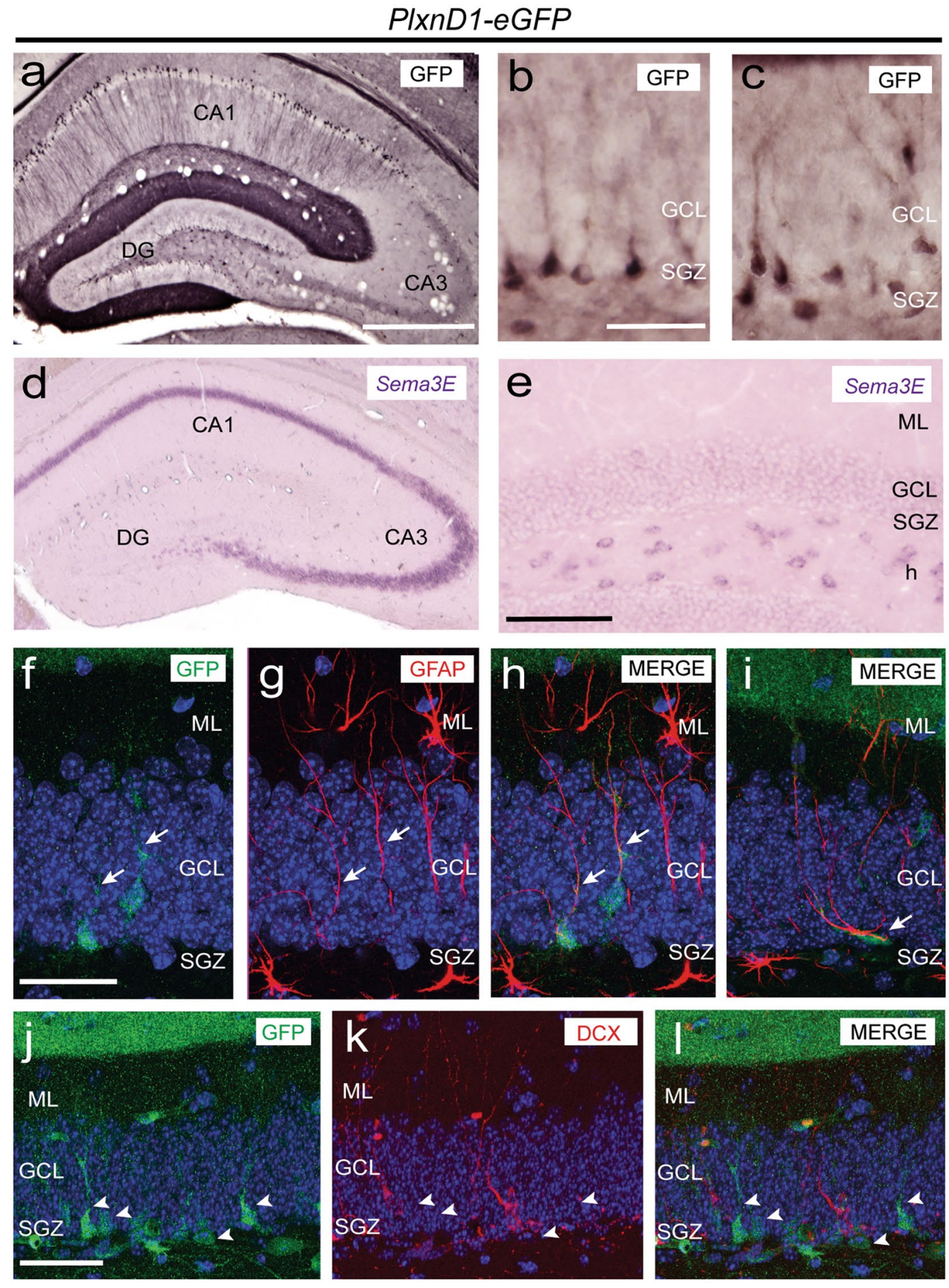

Figure 5. Low- (a) and high- (b,c) power photomicrographs illustrating GFP-positive neurons in the hippocampus (a) and dentate gyrus (b,c) of adult PlxnD1-eGFP mice. Note GFP labelling in subsets of pyramidal neurons CA1 and cells of hilus and subgranular zone. Low- (d) and high- (e) power photomicrographs illustrating the distribution of Sema3E mRNA in the hippocampus (d) and dentate gyrus (e) of adult wild-type mice. (f-i) Confocal immunofluorescence images for GFP (green) and GFAP (red) in the dentate gyrus of adult PlxnD1-eGFP mice. Note that all GFP-positive cells express GFAP marker (arrows). (j-l) Confocal immunofluorescence images for GFP (green) and DCX (red) in the dentate gyrus of adult PlxnD1$e G F P$ mice. Note that not all GFP-positive cells express DCX marker (arrowheads). Abbreviations as in Figs 1-4 and $\mathrm{ML}=$ molecular layer; $\mathrm{SGZ}=$ subgranular zone. Scale bars: $\mathbf{a}=500 \mu \mathrm{m}$ pertains to $\mathbf{d} ; \mathbf{b}=50 \mu \mathrm{m}$ pertains to $\mathbf{c} ; \mathbf{e}=150 \mu \mathrm{m} ; \mathbf{f}=50 \mu \mathrm{m}$ pertains to $(\mathbf{g}-\mathbf{i}) ; \mathbf{j}=50 \mu \mathrm{m}$ pertains to $(\mathbf{k}, \mathbf{l})$. 

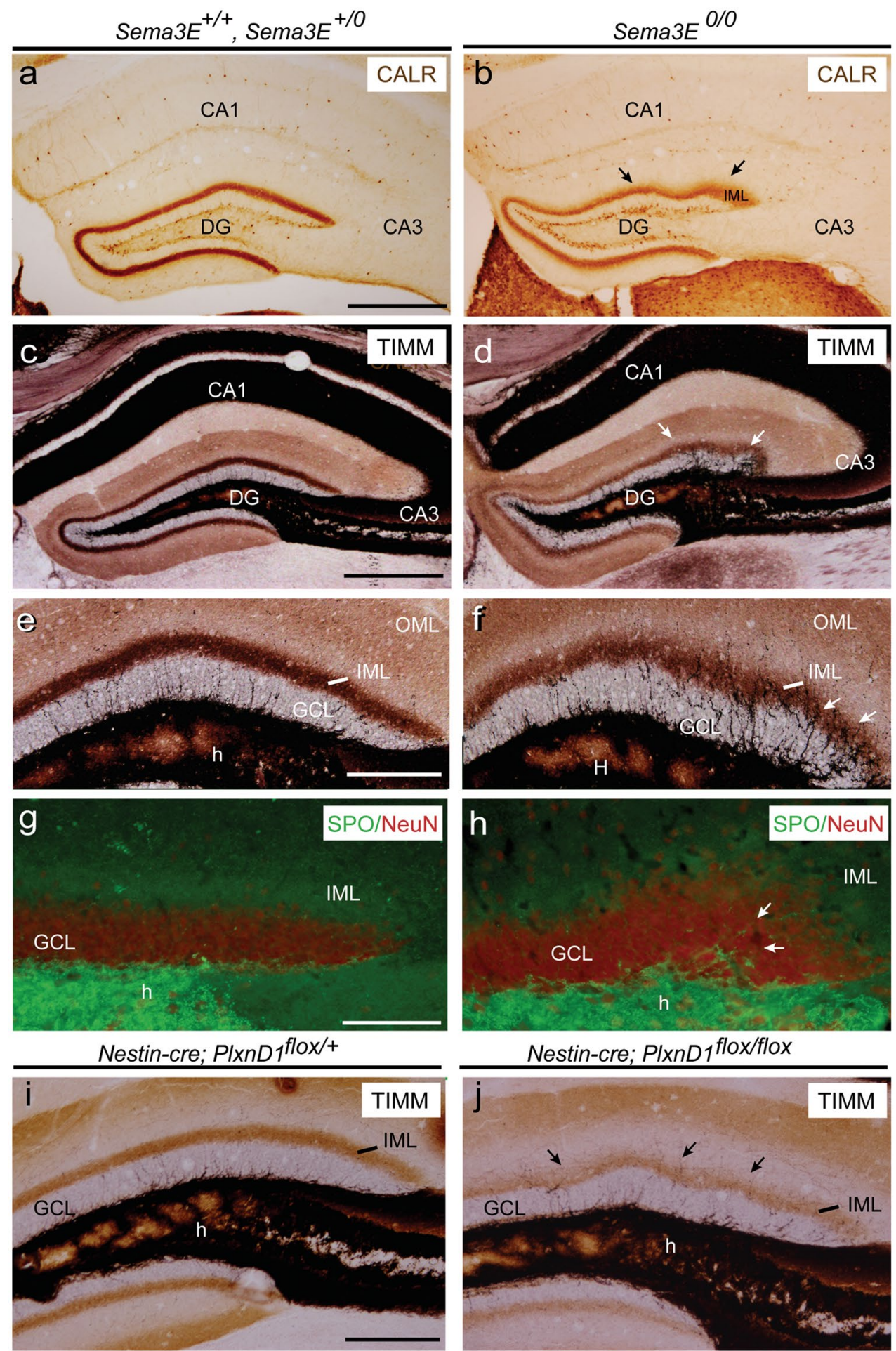

Figure 6. (a,b) Examples of $\alpha$-Calretinin immunostaining in the hippocampus proper and dentate gyrus of adult $\operatorname{Sema3} E^{+/+}$and $\operatorname{Sema}_{3} E^{+/ 0}(\mathbf{a})$ and $\operatorname{Sema} E^{0 / 0}(\mathbf{b})$ mice. Note the presence of several waves in the IML of the suprapyramidal blade (arrows in $\mathbf{b}$ ). (c-f) Photomicrographs illustrating the pattern of selenite-silver staining (TIMM) in Sema3 $E^{+/ 0}$ and Sema3 $E^{+/+}(\mathbf{c}, \mathbf{e})$ and $\operatorname{Sema}^{0 / 0}(\mathbf{d}, \mathbf{f})$ mice. Note the numerous ectopic mossy fibers crossing the granule cell layer entering the molecular layer of mutant mice (arrows in $\mathbf{d}, \mathbf{f})$. (g-h) Double immunolabeling of Synaptoporin (SPO, green) and NeuN (red) in the dentate gyrus of Sema3 $E^{+/+}$ and $\operatorname{Sema}^{+/ 0}(\mathbf{g})$ and $\operatorname{Sema} 3 E^{0 / 0}(\mathbf{h})$ mice showing ectopic mossy fibers crossing the granule cell layer in mutant mice (arrows in $\mathbf{h})$. (i-j) High-power photomicrographs illustrating the pattern of selenite-silver staining (TIMM) in control (Nestin-cre; PlxnD $1^{\text {flox/+ }}$ ) and PlexinD1-deficient (Nestin-cre; PlxnD $1^{\text {flox/flox }}$ ) mice. Abbreviations as in Figs $1-5$ and CALR $=$ calretinin; $S P O=$ synaptoporin. Scale bar: $\mathbf{a}=500 \mu \mathrm{m}$ pertains to $\mathbf{b}$ $\mathbf{c}=\mathbf{d}=500 \mu \mathrm{m} . \mathbf{e}=150 \mu \mathrm{m}$ pertains to $(\mathbf{f}, \mathbf{i}-\mathbf{j}) ; \mathbf{g}=\mathbf{h}=100 \mu \mathrm{m}$. 


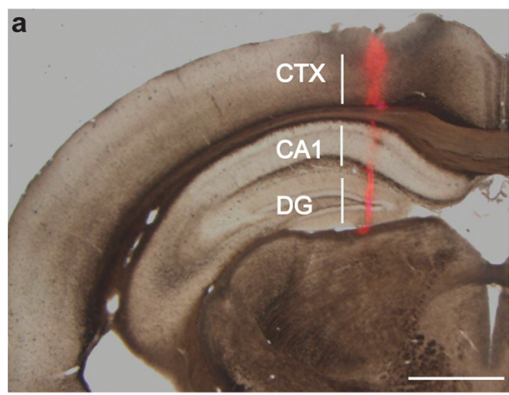

d

Up state duration

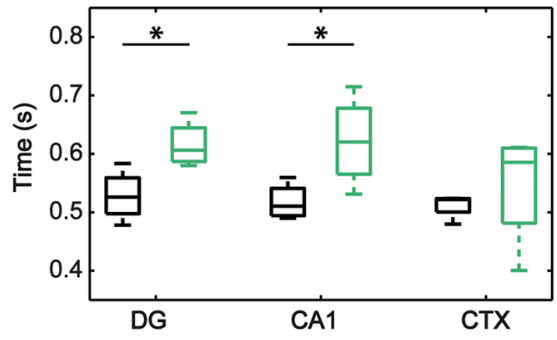

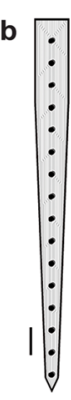

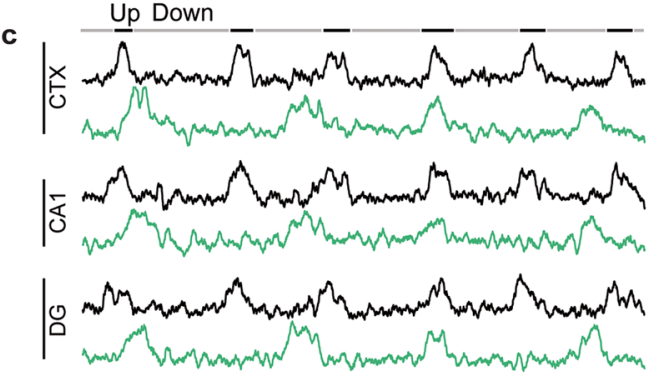

e

$\mathrm{X} 10^{3} \quad \mathrm{DG}$
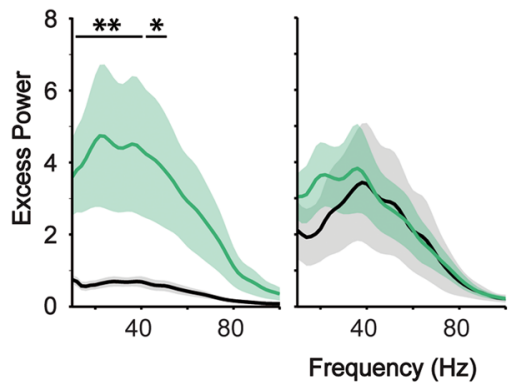

CTX

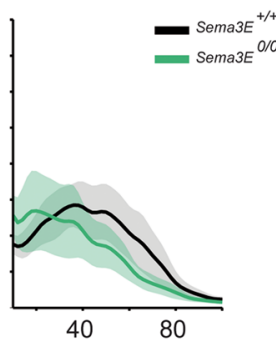

Figure 7. Alterations in the spontaneous oscillatory activity in Sema3 $E^{0 / 0}$ mice. (a) Representative half coronal section ( $2.0 \mathrm{~mm}$ posterior to Bregma, $1.0 \mathrm{~mm}$ lateral from midline) showing the track of a $1 \times 16$ multichannel recording probe covering the cerebral cortex (CTX) and hippocampus (CA1 and DG) of a mouse. Track reconstruction was accomplished following the tissue deposition of the DiI that was applied to probe prior to insertion. (b) Schematic representation of the 16-multichannel recording probe used to record neuronal activity. (c) Representative examples showing multi-unit activity traces $(200-1500 \mathrm{~Hz})$ simultaneously recorded in the cerebral cortex (CTX) and hippocampus (CA1 and DG) of a Sema3E $E^{+/+}$(black) and a Sema3E $E^{0 / 0}$ (green) mouse during slow oscillatory activity. No vertical scale because they are arbitrary units (see materials and Methods). (d) Up state durations recorded in the hippocampus (DG and CA1) and cerebral cortex (CTX) of Sema3 $E^{+/+}\left(\right.$black, $\mathrm{n}=4$ ) and $\operatorname{Sema}^{2} E^{0 / 0}$ (green, $\mathrm{n}=4$ ) mice. Box plots represent the first and third quartiles with the median depicted by the horizontal line within the box and extreme values shown by whiskers. (e) Average excess power (ratio between the mean power spectral density and the fit of the $1 / \mathrm{f}$ decay) during local field potential Up states recorded in the hippocampus (DG and CA1) and cerebral cortex (CTX) of Sema3 $E^{+/+}$ (black, $\mathrm{n}=4$ ) and $\operatorname{Sema}^{0 / 0}$ (green, $\mathrm{n}=4$ ) mice. Data expressed as mean \pm S.E.M (shadow). $* P<0.1$, $* * P<0.05$, Wilcoxon rank-sum test. Scale bar: $\mathbf{a}=1 \mathrm{~mm} ; \mathbf{b}=100 \mu \mathrm{m} ; \mathbf{c}=0.5 \mathrm{~s}$.

test), but not at the level of the overlying neocortex (Fig. 7c,d). Up states are generated by recurrent connectivity of local circuits and their duration is influenced by the balance between excitation and inhibition ${ }^{54-56}$ so elongated Up states have been considered a measure of increased excitability ${ }^{57,58}$. The longer duration of Up states at the level of dentate gyrus and CA1 areas of the hippocampus on Sema3 $E^{0 / 0}$ animals suggests an increased network excitability.

A fine balance between excitation and inhibition is also required for the generation of higher-frequency oscillatory components in both cortex and hippocampus ${ }^{54,59}$. In particular this gamma oscillatory activity, prominent in the aroused brain, has been implicated in higher-level cortical processes, such as sensory binding, storage of memories, and consciousness ${ }^{60}$ (see also $^{61}$ for review on hippocampal-associated gamma oscillations). Thus, to gain further insight into the possible dysregulation of the excitatory-inhibitory balance in $\operatorname{Sema} 3 E^{0 / 0}$ animals, we computed the power of the recorded oscillatory activity at higher frequencies $(10-100 \mathrm{~Hz})$ in our three target recording areas: neocortex, CA1 and dentate gyrus. As shown in Fig. 7e, the power of high-frequency oscillatory activity around the low-gamma range $(20-55 \mathrm{~Hz})$ was significantly greater in the dentate gyrus of $\operatorname{Sema} 3 E^{0 / 0}$ animals compared to Sema3E $E^{+/+}(\mathrm{p}<0.05$, Wilcoxon rank-sum test), while there were no significant differences at the level of either CA1 or cerebral cortex $(P>0.05$, Wilcoxon rank-sum test).

\section{Discussion}

Role of Sema3E/PlexinD1 signalling during the development of hippocampal connections. The participation of secreted semaphorins during cortical wiring has been reported in numerous studies (see Introduction for references). The present study expands current knowledge with the description of the particular actions of Sema3E and its receptor PlexinD1 during the formation of the EH connection. The entorhinal cortex plays a crucial role as a gateway, connecting the neocortex and the hippocampal formation. Layers II and III of the entorhinal cortex give rise to the perforant pathway, the main source of excitatory input to the hippocampus ${ }^{5-8}$. 
In the developing telencephalon, Sema3E/PlexinD1 functions have been described for Cajal-Retzius cell migration in neocortex ${ }^{41}$, as well as the development of subicular ${ }^{31,32}$ and striatal ${ }^{14}$ connections. However, their participation in the development of the EH connection had not been explored. Present results indicate that Sema3E is able to induce chemorepulsive actions on entorhinal, hippocampal and ventrolateral neocortical axons at E14.5 and E16.5. In a previous study ${ }^{32}$, Chauvet et al. reported that the addition of Sema3E to control cortical neurons from lateral (entorhinal-ventrolateral region in the present study) cortex (at E17.5) led to a 40\% decrease in mean axonal length. Similar effects on cortical axons were also described by Deck et al. at E14.5-E16.5 ${ }^{36}$. The present study, which indicates a similar chemorepulsive action on entorhinal axons growing in explant cultures and in confrontation assays (see Table 1) is in line with the above-mentioned study. In addition, our results demonstrate that this inhibition is PlexinD1 expression- dependent.

Our experiments also show chemorepulsive effects of Sema3E from E14.5 to E16.5 in hippocampal, entorhinal and ventrolateral neocortical axons, without chemoattractive responses. However, Bellon et al. reported Sema3E-induced chemoattraction on subicular neurons at E17.5 $5^{31}$. This chemoattractive effect is VEGFR2-dependent ${ }^{31}$. In our study, we were unable to observe VEGFR2 expression in subicular neurons between E14.5-E16.5 by histological methods (not shown). However, the inhibitory effects of Sema3E in subiculum decreased from E14.5 to E16.5 (P/D >1 from 75 to $60 \%$; with $\mathrm{P} / \mathrm{D}=1$ from 25 to $40 \%$ respectively). Thus, it is reasonable to consider a gradual shift in Sema3E-elicited effects in subicular neurons between E14.5 and E17.5 that might correlate with increased VEGFR2 expression in projecting subicular neurons. In addition, changes in semaphorin sensitivity in entorhinal cortex explants were also observed in Sema3F from E16.5 onwards, supported by the increasing Np2 labeling in entorhinal neurons between E14.5 and E16.5 in the region.

Previously reported data of Sema3E included a unique chemorepulsive effect on CA1-3 axons only at E14.5 without any effect in entorhinal axons prenatally or in CA1-3 axons between E16.5 and P0 ${ }^{30}$. In our study, Sema3E induced chemorepulsion in CA1-3 axons at E14.5 and E16.5, and in entorhinal axons at E14.5 with decreased effects at E16.5. In addition, ventrolateral cortical axons were also repelled by Sema3E at E14.5 and E16.5. In contrast, Sema3E is unable to trigger any apparent response in dorsal neocortex. Our data are strongly supported by previously published data $^{31}$ and also correlate with the temporal evolution of PlexinD1 expression in different subsets of projecting neurons, although a complete mechanistic understanding of these effects is not yet available. Lastly, in our study we described the almost complete absence of all three semaphorins (A, F and E) in the subiculum compared to other hippocampal subfields. As the EH connection crossed the subicular region (perforant pathway) during its navigation towards the hippocampus, it is reasonable to believe that this low semaphorin expression constitutes an 'axonal corridor' for entorhino-hippocampal axons to ensure appropriate establishment of the $\mathrm{EH}$ connections during embryonic development, as is also indicated for thalamo-cortical connections ${ }^{62}$. Our tracing experiments determined that the absence of Sema3E signaling did not essentially alter the basic pattern of termination of $\mathrm{EH}$ axons in vivo at early postnatal stages, although it caused minor targeting errors as also reported in Sema3 $A^{0 / 0}$ mice $^{30}$. These defects are not observed in adult hippocampus; they are probably resolved during postnatal synapse refinement as also reported in other axonal tracks of Sema3E-deficient mice ${ }^{31,32}$.

Adult functions of Sema3E/PlexinD1 signalling in hippocampus. The first description of $\operatorname{Sema3E^{0/0}}$ mice included reduced anxiety levels and moderately impaired spatial working memory ${ }^{32}$. It is well known that changes in mossy fiber distribution in different mouse strains correlated with deficits in spatial and non-spatial memory tasks ${ }^{63,64}$. Since Sema3E ${ }^{0 / 0}$ mutant mice showed changes in mossy fiber layer distribution, we cannot rule out the possibility that the described alterations also play a role in the impairment in the modest spatial tasks described in mutant mice ${ }^{32}$. The participation of several semaphorins (e.g., Sema3F $F^{33}$, Sema6A and Sema6B ${ }^{65}$ and their receptors (e.g., Neuropilin $2^{35}$, PlexinA $3^{34}$ or PlexinA $4^{65}$ ) in mossy fiber development has also been described. Our data led us to suggest the participation of Sema3E in mossy fiber development in combination with both other semaphorins and other families of guidance molecules (e.g., Ephrins ${ }^{66}$ ).

Zinc-positive mossy fiber terminals and SPO-positive synapses are ectopically located in the dentate iml and oml. These aberrant synapses have been described in several models of epilepsy (e.g. $\left.{ }^{49,50}\right)$. This opens the question of whether Sema3E and PlexinD1 expression levels could be modulated in seizures as happens with other class III semaphorins ${ }^{67-69}$ and neuropilins ${ }^{70-72}$. In our experiments, we did not find a convulsive phenotype or epileptogenic activity in Sema3 $E^{0 / 0}$ mice. However, we found an increased excitability in the dentate gyrus revealed by the longer Up states and increased high-frequency components, mainly in the gamma $(\approx 30-100 \mathrm{~Hz})$ band. Interestingly, the dentate gyrus is the gateway to centripetal synaptic connections originating in the entorhinal cortex $^{3}$. Moreover, neuronal activity of the entorhinal cortex has been demonstrated to modulate high-frequency gamma-like oscillatory activity at the level of the dentate gyrus ${ }^{73}$. Thus, it seems that the abnormal presence of ectopic mossy fibers and synaptic terminals found in the dentate gyrus of $S e m a 3 E^{0 / 0}$ mice might be related to the altered excitability present in these animals.

Furthermore, Sema3E and PlexinD1 expression levels could modulate synapse formation in the adult hippocampus. In this regard reduced levels of PlexinD1 decrease synapse density in neocortical neurons ${ }^{74}$ but increase dorsal thalamic input in striatal medium spiny neurons ${ }^{75}$. Our data suggesting an increase in zinc-positive sprouted fibers and boutons in the absence of Sema3E and PlxnD1 might corroborate the results reported by Ding in striatum ${ }^{75}$. Although the mechanism of this participation remains unknown, we should consider that the effects of Sema3E/PlexinD1 are mediated in particular cell types by modulating $\beta 1$-integrin function ${ }^{76}$ since mice lacking $\beta 1$-integrin-mediated signalling displayed several abnormalities very similar to those reported in our study ${ }^{77,78}$. Thus, it is reasonable to consider that Sema3E/PlexinD1 signalling modulates cell adhesion and synapse formation for subsets of granule cells in adult hippocampus.

The alteration in the lamination of the granule cells seems likely to be associated with local dysregulation of cell proliferation in the subgranular zone and/or with radial migration deficits. In this regard, high levels of Sema3E/PlexinD1 signalling decrease the proliferation of malignant (e.g. ${ }^{44,79,80}$ ) and non-malignant cells (e.g. $\left.{ }^{81}\right)$. 
Thus, we may hypothesize that an absence of Sema3E might induce increased proliferation of subsets of stem cells in the subgranular layer. Indeed, since PlexinD1 is not expressed by DCX-positive cells, effects of Sema3E could circumscribe the regulation of GFAP-positive, type-1 radial glia-like hippocampal stem cells ${ }^{82}$. Further studies are needed to determine whether these effects are limited to adult stages or are acquired during postnatal stages.

In conclusion, our study describes new roles of Sema3E/PlexinD1 signalling during development and in adult hippocampal formation, and clearly expands current knowledge of the proposed functions.

\section{Material and Methods}

Mice. Sema $3 E^{+/ 0}$ mice and $P l x n D 1^{\text {flox/flox }}$ mice were reported in ${ }^{38}$ and ${ }^{83}$ respectively. Mice were maintained in heterozygous genotype and experiments were performed using embryos of the different genotypes. Animals were genotyped by PCR, as previously described elsewhere ${ }^{38,83}$. Nestin-cre ${ }^{84}$ and CD1 mice were purchased from Jackson Laboratories (Bar Harbor, ME, USA). PlxnD1-eGFP mice were obtained from the Mutant Mouse Regional Resource Center (MMRRC; University of California, CA, USA). Females were mated overnight and the mating day confirmed by the presence of a vaginal plug was considered as embryonic day 0.5 (E0.5). The day of birth, the night between E19.5 to E20.5, was considered as postnatal day 0 (P0). Animals were maintained in a pathogen-free barrier facility at the University of Barcelona animal facility. All experiments were performed under the guidelines and protocols of the Ethical Committee for Animal Experimentation (CEEA) of the University of Barcelona, and the protocol for the use of animals in this study was reviewed and approved by the CEEA of the University of Barcelona (CEEA approval \#276/16 and 141/15).

Cell transfection, explant and organotypic slice culture procedures. Embryonic CA1-3, entorhinal, subicular, ventrolateral and dorsal neocortical explants from E14.5 and E16.5 embryos were dissected out and placed in three-dimensional hydrogel of rat tail Collagen $\mathrm{I}^{46}$. Explants were cultured at $37^{\circ} \mathrm{C}, 5 \% \mathrm{CO} 2$ and 95\% humidity in NeurobasalTM medium supplemented with B27 (Invitrogen) and glucose $(6.5 \mathrm{mg} / \mathrm{ml})$. After 2 days in vitro (DIV), genotypically identified cultures were paraformaldehyde-fixed and processed for BIII-tubulin (clone TUJ-1; Covance, BioLegend, San Diego, USA, Cat\#MMS-435P) immunostaining (see ${ }^{85}$ for details). For cell confrontation assays, transfected COS1 cell aggregates expressing SEAP (control AP vector), Sema3A-AP, Sema3F-AP or Sema3E-AP were confronted with the neural explants in hydrogel matrices. Quantification of TUJ1-positive growing axons was performed as indicated ${ }^{46}$. Proximal/distal ratio was calculated $(\mathrm{P} / \mathrm{D}<1$ chemorepulsion; $\mathrm{P} / \mathrm{D}=1$ radial growth and $\mathrm{P} / \mathrm{D}>1$ chemoattraction). Sema3A-AP, Sema3E-AP and Sema3F-AP plasmids were kindly provided by F. Mann. In addition, some experiments were conducted using commercial full-length cDNA clones Sema3A (MC205153), Sema3E (MC203244) and Sema3F (MC200862) purchased from OriGene Technologies (Rockville, MD, USA). As a second strategy, a growth cone collapse assay with entorhinal explants was also performed. Glass coverslips (12-mm $\varnothing$ ) were coated with Poly-L-Ornithine $(0.01 \%, 1 \mathrm{~h})$ and laminin $(2 \mu \mathrm{g} / \mathrm{ml}$, overnight). After washing, explants were placed in the same medium as above. After 3 DIV, their growth cones were visible and identifiable. To obtain the conditioned medium, COS1 cells were transfected with commercial Sema3E, Sema3A, Sema3F or SEAP expression vectors. Forty-eight hours after transfection, the medium was collected and 10 times concentrated in Millipore columns (Ultracel-30 membrane, Cat\#UFC903024). Explants were incubated with 15\% conditioned medium containing recombinant proteins or control media for $1 \mathrm{~h}$. Cultures were then fixed in $4 \%$ paraformaldehyde and stained with Phalloidin-TRITC (Sigma-Aldrich, St. Louis, MO, USA). For quantification, a total of 50-100 growth cones were analysed for each condition and percentage of collapsed axons was measured. For organotypic slice preparation, the brains of newborn Nestin-cre; PlxnD1 flox/+ (control, $\mathrm{n}=5$ ) and Nestin-cre; PlxnD $1^{\text {floxflox }}$ (mutant, $\mathrm{n}=5$ ) mice were dissected. Organotypic slices were prepared essentially as described ${ }^{47}$. After 7-10 days in vitro, the EH connection was traced with Byocitin ${ }^{47}$ and analysed.

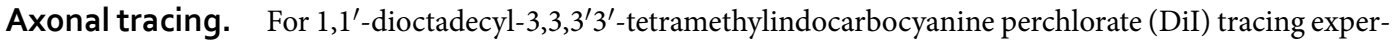
iments, postnatal mice were fixed with $4 \%$ paraformaldehyde dissolved in $0.1 \mathrm{M}$ phosphate buffer (pH 7.2-7.4). The brains were immersed in the same fixative solution at $4^{\circ} \mathrm{C} .300-\mu \mathrm{m}$ thick horizontal sections were obtained using a Vibratome. Sections were injected with a crystal of DiI (Molecular Probes, Eugene, OR, USA) in the entorhinal cortex using a thin metal needle under microscope control. After injection, sections were stored in fixative solution at room temperature for 3 to 10 days in darkness. Thereafter, sections were counterstained with bisbenzimide ( $5 \mu \mathrm{M}$, Sigma-Aldrich), mounted in Mowiol $^{\mathrm{TM}}$ and photodocumented.

Statistical processing. Statistical analysis of the obtained data (except electrophysiological studies) was performed using Bonferroni post hoc test (Multiple comparison test) using GraphPad Prism 6 (Mac OsX, Grahpad). Data are presented as mean \pm standard error of the mean (S.E.M).

In Vivo extracellular recordings. Surgical procedures. Four 12 -month-old Sema3 $E^{0 / 0}$ mice and their respective littermates $\left(S_{e m a} E^{+/+}\right)$were used for extracellular recordings. Anesthesia was induced by the intraperitoneal administration of a mixture of ketamine $(50 \mathrm{mg} / \mathrm{kg})$ and medetomidine $(1.3 \mathrm{mg} / \mathrm{kg})$ and maintained, after tracheotomy, by the constant infusion of isoflurane (1\%) in oxygen (100\%). Atropine (0.3 mg/kg) and methylprednisolone $(30 \mathrm{mg} / \mathrm{kg}$ ) were administered subcutaneously to avoid respiratory secretions and prevent the appearance of edema. To study evoked epileptic discharges, kainic acid $(10 \mathrm{mg} / \mathrm{kg})$ was administered intraperitoneally. Body temperature was constantly monitored and kept at $37^{\circ} \mathrm{C}$ by means of a thermal blanket (RWD Life Science, San Diego, CA, USA). Once stabilized, animals were placed on a stereotaxic frame (SR-6M, Narishige, London, UK) and a craniotomy was performed over the target area of the hippocampus (2.0 mm posterior to bregma, $1.0 \mathrm{~mm}$ lateral from midline $\mathrm{e}^{86}$ ). 
Extracellular recordings. Extracellular activity was recorded by means of 16-channel multielectrode probes (100 $\mu \mathrm{m}$ spacing, E16-100-S1-L6, Atlas Neuroengineering, Leuven, Belgium) previously marked with DiI for anatomical reconstruction, covering the DG and CA1 areas of the hippocampus and the overlying cerebral cortex. The signal was amplified (Multichannel systems (Germany)), digitized at $20 \mathrm{kHz}$ and fed into a computer via a digitizer interface (CED 1401 interface and Spike2 software; Cambridge Electronic Design).

Data analysis. Up and Down detection was done as previously described ${ }^{87}$. Briefly, the slow oscillation envelope, the envelope of the variance of the gamma-filtered local field potential (LFP) and the estimated multi-unit activity, were linearly combined to generate a time series where Up and Down states were singled out by setting a threshold. After detection, the mean duration of Up and Down states was calculated and Up state high-frequency components were analysed using Welch's power spectrum density methods. Epileptic-like events were detected from the envelope of the variance of the raw LFP signal band-pass filtered between $3-6 \mathrm{~Hz}$ following previously described methods ${ }^{88}$. Data were analysed using the Wilcoxon rank-sum non-parametric statistical hypothesis testusing GraphPad Prism 6 (Mac OsX, Grahpad).

Availability of materials and data. All data generated or analysed during this study are included in this published article (and its Supplementary information files).

\section{References}

1. Bird, C. M. The role of the hippocampus in recognition memory. Cortex 93, 155-165 (2017).

2. Eichenbaum, H. The role of the hippocampus in navigation is memory. J Neurophysiol 117, 1785-1796 (2017).

3. van Groen, T., Miettinen, P. \& Kadish, I. The entorhinal cortex of the mouse: organization of the projection to the hippocampal formation. Hippocampus 13, 133-149 (2003).

4. Taupin, P. The hippocampus: neurotransmission and plasticity in the nervous system (Nova Biomedical Books, 2007).

5. Amaral, D. G. \& Witter, M. P. The three-dimensional organization of the hippocampal formation: a review of anatomical data. Neuroscience 31, 571-591 (1989).

6. Witter, M. P., Van Hoesen, G. W. \& Amaral, D. G. Topographical organization of the entorhinal projection to the dentate gyrus of the monkey. J Neurosci 9, 216-228 (1989).

7. Burwell, R. D., Witter, M. P. \& Amaral, D. G. Perirhinal and postrhinal cortices of the rat: a review of the neuroanatomical literature and comparison with findings from the monkey brain. Hippocampus 5, 390-408 (1995).

8. Isaacson, R. L. \& Pribram, K. H. The Hippocampus. (Plenum Press, 1975).

9. Zhou, W., Raisman, G. \& Zhou, C. Transplanted embryonic entorhinal neurons make functional synapses in adult host hippocampus. Brain Res 788, 202-206 (1998).

10. Davies, S. J., Field, P. M. \& Raisman, G. Embryonic tissue induces growth of adult axons from myelinated fiber tracts. Exp Neurol 145, 471-476 (1997).

11. Woodhams, P. L., Kawano, H. \& Raisman, G. The OM series of terminal field-specific monoclonal antibodies demonstrate reinnervation of the adult rat dentate gyrus by embryonic entorhinal transplants. Neuroscience 46, 71-82 (1992).

12. Li, D., Field, P. M., Yoshioka, N. \& Raisman, G. Axons regenerate with correct specificity in horizontal slice culture of the postnatal rat entorhino-hippocampal system. Eur J Neurosci 6, 1026-1037 (1994)

13. Li, D., Field, P. M. \& Raisman, G. Connectional specification of regenerating entorhinal projection neuron classes cannot be overridden by altered target availability in postnatal organotypic slice co-culture. Exp Neurol 142, 151-160 (1996).

14. Li, D., Field, P. M. \& Raisman, G. Failure of axon regeneration in postnatal rat entorhinohippocampal slice coculture is due to maturation of the axon, not that of the pathway or target. Eur J Neurosci 7, 1164-1171 (1995).

15. Del Rio, J. A. et al. Differential survival of Cajal-Retzius cells in organotypic cultures of hippocampus and neocortex. J Neurosci 16, 6896-6907 (1996).

16. Del Rio, J. A. et al. A role for Cajal-Retzius cells and reelin in the development of hippocampal connections. Nature 385, 70-74 (1997).

17. Savaskan, N. E. et al. Entorhinal cortex lesion studied with the novel dye fluoro-jade. Brain Res 864, 44-51 (2000).

18. Super, H., Martinez, A., Del Rio, J. A. \& Soriano, E. Involvement of distinct pioneer neurons in the formation of layer-specific connections in the hippocampus. J Neurosci 18, 4616-4626 (1998).

19. Borrell, V., Ruiz, M., Del Rio, J. A. \& Soriano, E. Development of commissural connections in the hippocampus of reeler mice: evidence of an inhibitory influence of Cajal-Retzius cells. Exp Neurol 156, 268-282 (1999).

20. Frotscher, M. \& Heimrich, B. Formation of layer-specific fiber projections to the hippocampus in vitro. Proceedings of the National Academy of Sciences of the United States of America 90, 10400-10403 (1993).

21. Skutella, T. \& Nitsch, R. New molecules for hippocampal development. Trends Neurosci 24, 107-113 (2001).

22. Savaskan, N. E. \& Nitsch, R. Molecules involved in reactive sprouting in the hippocampus. Rev Neurosci 12, 195-215 (2001).

23. Deller, T., Haas, C. A. \& Frotscher, M. Sprouting in the hippocampus after entorhinal cortex lesion is layer- specific but not translaminar: which molecules may be involved? Restor Neurol Neurosci 19, 159-167 (2001).

24. Yu, H. H. \& Kolodkin, A. L. Semaphorin signaling: a little less per-plexin. Neuron 22, 11-14 (1999).

25. Bagri, A. \& Tessier-Lavigne, M. Neuropilins as Semaphorin receptors: in vivo functions in neuronal cell migration and axon guidance. Adv Exp Med Biol 515, 13-31 (2002).

26. Kolodkin, A. L. \& Tessier-Lavigne, M. Mechanisms and molecules of neuronal wiring: a primer. Cold Spring Harb Perspect Biol 3, (2011).

27. Steup, A. et al. Semaphorin D acts as a repulsive factor for entorhinal and hippocampal neurons. Eur J Neurosci 11, 729-734 (1999).

28. Chedotal, A. et al. Semaphorins III and IV repel hippocampal axons via two distinct receptors. Development 125, 4313-4323 (1998).

29. Rubio, S. E. et al. Semaphorin 3C is not required for the establishment and target specificity of the GABAergic septohippocampal pathway in vitro. Eur J Neurosci 34, 1923-1933 (2011).

30. Pozas, E. et al. Age-dependent effects of secreted Semaphorins 3A, 3F, and 3E on developing hippocampal axons: in vitro effects and phenotype of Semaphorin 3A (-/-) mice. Mol Cell Neurosci 18, 26-43 (2001).

31. Bellon, A. et al. VEGFR2 (KDR/Flk1) signaling mediates axon growth in response to semaphorin $3 \mathrm{E}$ in the developing brain. Neuron 66, 205-219 (2010).

32. Chauvet, S. et al. Gating of Sema3E/PlexinD1 signaling by neuropilin-1 switches axonal repulsion to attraction during brain development. Neuron 56, 807-822 (2007).

33. Sahay, A., Molliver, M. E., Ginty, D. D. \& Kolodkin, A. L. Semaphorin 3F is critical for development of limbic system circuitry and is required in neurons for selective CNS axon guidance events. J Neurosci 23, 6671-6680 (2003).

34. Cheng, H. J. et al. Plexin-A3 mediates semaphorin signaling and regulates the development of hippocampal axonal projections. Neuron 32, 249-263 (2001). 
35. Chen, H. et al. Neuropilin-2 regulates the development of selective cranial and sensory nerves and hippocampal mossy fiber projections. Neuron 25, 43-56 (2000).

36. Deck, M. et al. Pathfinding of corticothalamic axons relies on a rendezvous with thalamic projections. Neuron 77, 472-484 (2013).

37. Kim, J., Oh, W. J., Gaiano, N., Yoshida, Y. \& Gu, C. Semaphorin 3E-Plexin-D1 signaling regulates VEGF function in developmental angiogenesis via a feedback mechanism. Genes Dev 25, 1399-1411 (2011).

38. Gu, C. et al. Semaphorin 3E and plexin-D1 control vascular pattern independently of neuropilins. Science 307, 265-268 (2005).

39. Chauvet, S., Burk, K. \& Mann, F. Navigation rules for vessels and neurons: cooperative signaling between VEGF and neural guidance cues. Cell Mol Life Sci, (2013).

40. Oh, W. J. \& Gu, C. The role and mechanism-of-action of Sema3E and Plexin-D1 in vascular and neural development. Semin Cell Dev Biol 24, 156-162 (2013).

41. Bribian, A. et al. Sema3E/PlexinD1 regulates the migration of hem-derived Cajal-Retzius cells in developing cerebral cortex. Nat Commun 5, 4265 (2014).

42. Sharma, A., Pollett, M. A., Plant, G. W. \& Harvey, A. R. Changes in mRNA expression of class 3 semaphorins and their receptors in the adult rat retino-collicular system after unilateral optic nerve injury. Invest Ophthalmol Vis Sci 53, 8367-8377 (2012).

43. Lai Wing Sun, K., Correia, J. P. \& Kennedy, T. E. Netrins: versatile extracellular cues with diverse functions. Development 138, 2153-2169 (2011).

44. Sabag, A. D. et al. Semaphorin-3D and semaphorin-3E inhibit the development of tumors from glioblastoma cells implanted in the cortex of the brain. PloS one 7, e42912 (2012).

45. Roodink, I. et al. Semaphorin 3E expression correlates inversely with Plexin D1 during tumor progression. Am J Pathol 173, 1873-1881 (2008).

46. Gil, V. \& del Rio, J. A. Analysis of axonal growth and cell migration in 3D hydrogel cultures of embryonic mouse CNS tissue. Nat Protoc 7, 268-280 (2012).

47. del Rio, J. A. \& Soriano, E. Regenerating cortical connections in a dish: the entorhino-hippocampal organotypic slice co-culture as tool for pharmacological screening of molecules promoting axon regeneration. Nat Protoc 5, 217-226 (2010).

48. Watakabe, A., Ohsawa, S., Hashikawa, T. \& Yamamori, T. Binding and complementary expression patterns of semaphorin $3 \mathrm{E}$ and plexin D1 in the mature neocortices of mice and monkeys. J Comp Neurol 499, 258-273 (2006).

49. Mitchell, J., Gatherer, M. \& Sundstrom, L. E. Aberrant Timm-stained fibres in the dentate gyrus following tetanus toxin-induced seizures in the rat. Neuropathol Appl Neurobiol 22, 129-135 (1996).

50. Hamani, C., Paulo, I. \& Mello, L. E. Neo-Timm staining in the thalamus of chronically epileptic rats. Braz J Med Biol Res 38, $1677-1682(2005)$.

51. Steriade, M., Nunez, A. \& Amzica, F. A novel slow $(<1 \mathrm{~Hz})$ oscillation of neocortical neurons in vivo: depolarizing and hyperpolarizing components. J Neurosci 13, 3252-3265 (1993).

52. Ruiz-Mejias, M. et al. Overexpression of Dyrk1A, a Down Syndrome Candidate, Decreases Excitability and Impairs Gamma Oscillations in the Prefrontal Cortex. J Neurosci 36, 3648-3659 (2016).

53. Timofeev, I., Contreras, D. \& Steriade, M. Synaptic responsiveness of cortical and thalamic neurones during various phases of slow sleep oscillation in cat. J Physiol 494(Pt 1), 265-278 (1996)

54. Compte, A. et al. Spontaneous high-frequency $(10-80 \mathrm{~Hz})$ oscillations during up states in the cerebral cortex in vitro. J Neurosci $\mathbf{2 8}$, 13828-13844 (2008).

55. Contreras, D., Timofeev, I. \& Steriade, M. Mechanisms of long-lasting hyperpolarizations underlying slow sleep oscillations in cat corticothalamic networks. J Physiol 494(Pt 1), 251-264 (1996).

56. Sanchez-Vives, M. V. \& McCormick, D. A. Cellular and network mechanisms of rhythmic recurrent activity in neocortex. Nat Neurosci 3, 1027-1034 (2000).

57. Gibson, J. R., Bartley, A. F., Hays, S. A. \& Huber, K. M. Imbalance of neocortical excitation and inhibition and altered UP states reflect network hyperexcitability in the mouse model of fragile X syndrome. J Neurophysiol 100, 2615-2626 (2008).

58. Huo, Q. et al. Prefrontal Cortical GABAergic Dysfunction Contributes to Aberrant UP-State Duration in APP Knockout Mice. Cereb Cortex, (2016).

59. Csicsvari, J., Jamieson, B., Wise, K. D. \& Buzsaki, G. Mechanisms of gamma oscillations in the hippocampus of the behaving rat. Neuron 37, 311-322 (2003).

60. Buzsaki, G. \& Wang, X. J. Mechanisms of gamma oscillations. Annu Rev Neurosci 35, 203-225 (2012).

61. Mann, E. O. \& Paulsen, O. Mechanisms underlying gamma (' $40 \mathrm{~Hz}$ ') network oscillations in the hippocampus-a mini-review. Prog Biophys Mol Biol 87, 67-76 (2005)

62. Maroof, A. M. \& Anderson, S. A. Off on a tangent: thalamocortical axons traverse a permissive corridor across the basal telencephalon. Neuron 50, 185-188 (2006).

63. Crusio, W. E., Schwegler, H. \& Brust, I. Covariations between hippocampal mossy fibres and working and reference memory in spatial and non-spatial radial maze tasks in mice. Eur J Neurosci 5, 1413-1420 (1993).

64. Schwegler, H., Crusio, W. E. \& Brust, I. Hippocampal mossy fibers and radial-maze learning in the mouse: a correlation with spatial working memory but not with non-spatial reference memory. Neuroscience 34, 293-298 (1990).

65. Tawarayama, H., Yoshida, Y., Suto, F., Mitchell, K. J. \& Fujisawa, H. Roles of semaphorin-6B and plexin-A2 in lamina-restricted projection of hippocampal mossy fibers. J Neurosci 30, 7049-7060 (2010).

66. Martinez, A. \& Soriano, E. Functions of ephrin/Eph interactions in the development of the nervous system: emphasis on the hippocampal system. Brain Res Brain Res Rev 49, 211-226 (2005).

67. Yang, J. et al. Genetic background regulates semaphorin gene expression and epileptogenesis in mouse brain after kainic acid status epilepticus. Neuroscience 131, 853-869 (2005).

68. Barnes, G., Puranam, R. S., Luo, Y. \& McNamara, J. O. Temporal specific patterns of semaphorin gene expression in rat brain after kainic acid-induced status epilepticus. Hippocampus 13, 1-20 (2003)

69. Sahay, A. et al. Secreted semaphorins modulate synaptic transmission in the adult hippocampus. J Neurosci 25, 3613-3620 (2005).

70. Gant, J. C. et al. Decreased number of interneurons and increased seizures in neuropilin 2 deficient mice: implications for autism and epilepsy. Epilepsia 50, 629-645 (2009).

71. De Winter, F., Holtmaat, A. J. \& Verhaagen, J. Neuropilin and class 3 semaphorins in nervous system regeneration. Adv Exp Med Biol $\mathbf{5 1 5}, 115-139(2002)$

72. Shimakawa, S. et al. Neuropilin-2 is overexpressed in the rat brain after limbic seizures. Brain Res 956, 67-73 (2002).

73. Sullivan, D. et al. Relationships between hippocampal sharp waves, ripples, and fast gamma oscillation: influence of dentate and entorhinal cortical activity. J Neurosci 31, 8605-8616 (2011).

74. Wang, F., Eagleson, K. L. \& Levitt, P. Positive regulation of neocortical synapse formation by the Plexin-D1 receptor. Brain Res 1616, 157-165 (2015)

75. Ding, J. B., Oh, W. J., Sabatini, B. L. \& Gu, C. Semaphorin 3E-Plexin-D1 signaling controls pathway-specific synapse formation in the striatum. Nat Neurosci 15, 215-223 (2012).

76. Choi, Y. I. et al. Dynamic control of beta1 integrin adhesion by the plexinD1-sema3E axis. Proceedings of the National Academy of Sciences of the United States of America 111, 379-384 (2014).

77. Bhattacharya, D. et al. Impaired ILK Function Is Associated with Deficits in Hippocampal Based Memory and Synaptic Plasticity in a FASD Rat Model. PloS one 10, e0135700 (2015). 
78. Beggs, H. E. et al. FAK deficiency in cells contributing to the basal lamina results in cortical abnormalities resembling congenital muscular dystrophies. Neuron 40, 501-514 (2003).

79. Chen, H. et al. Epigenetically downregulated Semaphorin 3E contributes to gastric cancer. Oncotarget 6, 20449-20465 (2015).

80. Casazza, A. et al. Tumour growth inhibition and anti-metastatic activity of a mutated furin-resistant Semaphorin $3 E$ isoform. EMBO Mol Med 4, 234-250 (2012).

81. Movassagh, H. et al. Neuronal chemorepellent Semaphorin 3E inhibits human airway smooth muscle cell proliferation and migration. J Allergy Clin Immunol 133, 560-567 (2014).

82. Kempermann, G., Song, H. \& Gage, F. H. Neurogenesis in the Adult Hippocampus. Cold Spring Harb Perspect Biol 7, a018812 (2015).

83. Fukuhara, K. et al. Specificity of monosynaptic sensory-motor connections imposed by repellent Sema3E-PlexinD1 signaling. Cell Rep 5, 748-758 (2013).

84. Dubois, N. C., Hofmann, D., Kaloulis, K., Bishop, J. M. \& Trumpp, A. Nestin-Cre transgenic mouse line Nes-Cre1 mediates highly efficient Cre/loxP mediated recombination in the nervous system, kidney, and somite-derived tissues. Genesis 44, 355-360 (2006).

85. Simo, S. et al. Reelin induces the detachment of postnatal subventricular zone cells and the expression of the Egr-1 through Erk1/2 activation. Cereb Cortex 17, 294-303 (2007).

86. Paxinos, G. \& Franklin, K. B. J. The mouse brain in stereotaxic coordinates. Compact 2nd edn, (Elsevier Academic Press, 2004).

87. Castano-Prat, P., Perez-Zabalza, M., Perez-Mendez, L., Escorihuela, R. M. \& Sanchez-Vives, M. V. Slow and Fast Neocortical Oscillations in the Senescence-Accelerated Mouse Model SAMP8. Front Aging Neurosci 9, 141 (2017).

88. Colic, S., Wither, R. G., Zhang, L., Eubanks, J. H. \& Bardakjian, B. L. Characterization of seizure-like events recorded in vivo in a mouse model of Rett syndrome. Neural Netw 46, 109-115 (2013).

\section{Acknowledgements}

The authors thank Tom Yohannan for editorial advice and M. Segura-Feliu for technical assistance. The authors also thank Prof. Yutaka Yoshida (Division of Developmental Biology, Cincinnati Children's Hospital Medical Center, Cincinnati, Ohio, USA) and Prof. Fanny Mann (Developmental Biology Institute of Marseille Luminy, CNRS UMR 6216, University of Mediterranee, Marseille, France) for providing the PlxnD $1^{\text {flox/flox }}$ and Sema3E $E^{+/ o}$ mice respectively and for critical reading of the manuscript. We also thank Profs. Alex L. Kolodkin and Alain Chedotal for providing reagents. This research was supported by grants from the Spanish Ministry of Economy and Competitiveness (MEICO) (BFU2015-67777-R and TEC2015-72718-EXP); the Spanish Prion Network (Prionet Spain, AGL2015-71764-REDT); the Generalitat de Catalunya (SGR2014-1218); CIBERNED (PRY201602) and PRY14-114) and La Marató de TV3 (20143410) to JADR and a grant from MINECO (SAF201342445-R) to ES. JMGV was supported by the Prometeo grant PROMETEOII/2014/075. MVSV was supported by FLAGERA-PCIN-2015-162-C02-01, BFU2014-52467-R (MINECO) and CERCA (Generalitat de Catalunya). AM was supported by a fellowship from MINECO (BES-2013-062854). VG was supported by a Juan de la Cierva post-doctoral fellowship from MINECO (JCI-2012-14356).

\section{Author Contributions}

A.M. and V.G. performed most of the experiments. J.P. contributed in selenite-silver staining, E.S. supervised the explant cultures experiments. M.D. and M.V.S. developed the in vivo extracellular recordings and M.C.G. and J.M.G. performed the electron microscopy experiment. A.M., V.G. and J.A.d.R. designed the project and wrote the manuscript. All authors reviewed the manuscript.

\section{Additional Information}

Supplementary information accompanies this paper at https://doi.org/10.1038/s41598-018-19794-0.

Competing Interests: The authors declare that they have no competing interests.

Publisher's note: Springer Nature remains neutral with regard to jurisdictional claims in published maps and institutional affiliations.

(c) (i) Open Access This article is licensed under a Creative Commons Attribution 4.0 International

License, which permits use, sharing, adaptation, distribution and reproduction in any medium or format, as long as you give appropriate credit to the original author(s) and the source, provide a link to the Creative Commons license, and indicate if changes were made. The images or other third party material in this article are included in the article's Creative Commons license, unless indicated otherwise in a credit line to the material. If material is not included in the article's Creative Commons license and your intended use is not permitted by statutory regulation or exceeds the permitted use, you will need to obtain permission directly from the copyright holder. To view a copy of this license, visit http://creativecommons.org/licenses/by/4.0/.

(c) The Author(s) 2018 